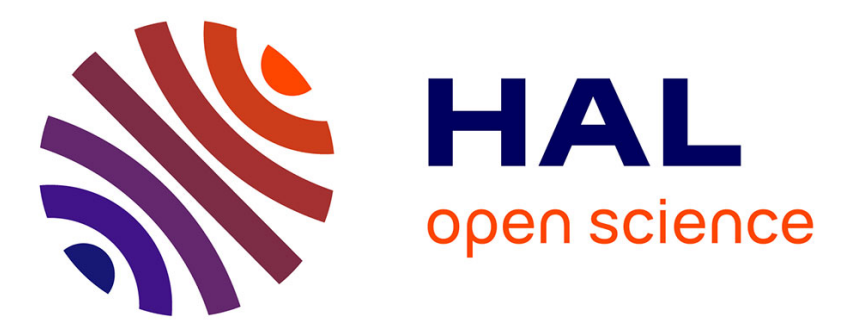

\title{
Optimization of the Cut-Off Rate of Generalized Spatial Modulation with Transmit Precoding
}

Nemanja Stefan Perovic, Peng Liu, Jiri Blumenstein, Marco Di Renzo, Andreas Springer

\section{- To cite this version:}

Nemanja Stefan Perovic, Peng Liu, Jiri Blumenstein, Marco Di Renzo, Andreas Springer. Optimization of the Cut-Off Rate of Generalized Spatial Modulation with Transmit Precoding. IEEE Transactions on Communications, 2018, 10.1109/TCOMM.2018.2833548 . hal-01880364

\section{HAL Id: hal-01880364 https://hal.science/hal-01880364}

Submitted on 7 Jul 2020

HAL is a multi-disciplinary open access archive for the deposit and dissemination of scientific research documents, whether they are published or not. The documents may come from teaching and research institutions in France or abroad, or from public or private research centers.
L'archive ouverte pluridisciplinaire HAL, est destinée au dépôt et à la diffusion de documents scientifiques de niveau recherche, publiés ou non, émanant des établissements d'enseignement et de recherche français ou étrangers, des laboratoires publics ou privés. 


\title{
Optimization of the Cut-Off Rate of Generalized Spatial Modulation with Transmit Precoding
}

\author{
Nemanja Stefan Perović, Peng Liu, Member, IEEE, Jiri Blumenstein, Member, IEEE, Marco Di Renzo, Senior \\ Member, IEEE, and Andreas Springer, Member, IEEE
}

\begin{abstract}
Spatial modulation (SM) and generalized spatial modulation (GSM) are emerging multiple input multiple output (MIMO) schemes that use transmitter (TX) antenna switching for data transmission. Their operating principle makes optimization of channel capacity and mutual information usually more difficult than for conventional MIMO schemes which are not based on antenna switching. We propose to use channel cut-off rate as a relevant and more tractable metric for performance optimization of SM/GSM systems, as it constitutes a practical lower-bound of channel capacity. In particular, we propose four TX precoding schemes for increasing the cut-off rate of SM/GSM systems. We show that those TX precoding schemes which are designed for increasing array gain provide the largest improvement of cutoff rate for low signal-to-noise ratio (SNR). On the other hand, the TX precoding schemes that are designed for increasing the minimum Euclidean distance of GSM symbols are more suitable for application to medium to high SNR setups and correlated channels. The proposed precoding schemes are shown to be able to enhance mutual information, and the gain is shown to be of the same order of magnitude as the gain of the corresponding channel cut-off rate.
\end{abstract}

Index Terms-Channel cut-off rate, channel capacity, generalized spatial modulation (GSM), mutual information, optimization, TX precoding.

\section{INTRODUCTION}

Among the many multiple input multiple output (MIMO) schemes that exist in the literature, spatial modulation (SM) and generalized spatial modulation (GSM) have attracted a lot of research interest during the last decade [1], [2]. In the last

Nemanja Stefan Perović is with the Institute for Communications Engineering and RF-Systems, Johannes Kepler University Linz, 4040 Linz, Austria (e-mail: nemanja_stefan.perovic@jku.at). Andreas Springer is with the Christian Doppler Laboratory for Digitally Assisted RF Transceivers for Future Mobile Communications, Institute for Communications Engineering and RF-Systems, Johannes Kepler University Linz, 4040 Linz, Austria (e-mail: andreas.springer@jku.at). Peng Liu is with Arralis Ltd., Tierney Building UL, Castletroy, Limerick, Ireland (IRL) (e-mail: peng.liu@ arralis.com). The work of Peng Liu and Andreas Spriger was supported in part by the Austrian COMET-K2 Programme, Linz Center of Mechatronics, funded by the Austrian Federal Government and the Federal State of Upper Austria.

Jiri Blumenstein is with the Department of Radioeletronics, Brno University of Technology (BUT), 60190 Brno, Czech Republic (e-mail: blumenstein@feec.vutbr.cz). His research was financed by the Czech Science Foundation, Project No. 17-27068S, and by the National Sustainability Program under grant LO1401. For his research, the infrastructure of the SIX Center was used.

Marco Di Renzo is with the Laboratoire des Signaux et Systèmes, CNRS, CentraleSupélec, Univ Paris Sud, Université Paris-Saclay, 3 rue Joliot Curie, Plateau du Moulon, 91192 Gif-sur-Yvette, France (e-mail marco.direnzo@12s.centralesupelec.fr). His work is supported in part by the Agence Nationale de la Recherche Scientifique (ANR) through the research project SpatialModulation (Société de l'Information et de la Communication - Action Plan 2015). years, in particular, many schemes that enhance and optimize the performance of SM/GSM systems have been proposed. Most of them are focused on optimizing error performance, while only a few of them are concerned with optimization of channel capacity and mutual information. This is due to the inherent complexity of formulating the channel capacity of SM/GSM systems in a mathematically tractable manner that is suitable for system optimization [3]. This is, on the other hand, relatively simpler to do for error probability. To overcome this issue, we propose to use channel cut-off rate, as a practical surrogate of channel capacity, for optimizing capacity and mutual information of SM/GSM systems [4].

Channel cut-off rate constitutes an upper-bound of the codeword error probability of a communication system over the ensemble of random binary channel codes, and, thus, it is independent of the specific code being used. The main motivation for proposing it as a substitute of channel capacity for optimizing the performance of SM/GSM systems originates from James Massey, who suggested that, as a rule of thumb, channel cut-off rate is the practical upper-limit on the code rate for reliable communications, whereas channel capacity is the theoretical upper-limit [4]. Channel cut-off rate is a widely accepted and sensible performance indicator for comparing channels for which a coding scheme with finite complexity is used [5]. In the context of optimizing the design of SM/GSM systems, relying on channel cut-off rate instead of channel capacity has two main advantages: i) optimization of the modulator is independent of the specific channel code being used [6]; and ii) there is no need to deal with the intractable mathematical expression of the channel capacity of SM/GSM [3].

In mathematical terms, the relation between channel cut-off rate $R_{0}$ and codeword error probability $P_{e}$ can be formulated as $P_{e} \leq e^{-n\left(R_{0}-R\right)}$, where $n$ is the number of times that the channel is used for transmitting a codeword (i.e., the codeword length), and $R$ is the information rate in bits per channel use (i.e., the binary code rate) [7], [8]. For very long code sequences (i.e., $n \rightarrow \infty), P_{e}$ can approach zero, i.e., it becomes arbitrarily small, as long as $R<R_{0}$. Hence, channel cut-off rate can be interpreted as a practical lower-bound of channel capacity [9].

In SM-MIMO systems, two data streams are transmitted one in the conventional in-phase and quadrature (IQ) domain by employing, e.g., $M$-PSK or $M$-QAM modulation, and the other one in the so-called spatial domain, i.e., by selecting and activating one out of $N_{t}$ antennas that are available at the transmitter (TX) [1], [2]. A generalization of SM consists 
either of activating more than one TX antenna per time slot while still transmitting a single IQ stream [10], or of activating multiple TX antennas and transmitting more than one IQ stream [11], [12]. This generalized scheme is referred to as GSM and it provides higher data rates than SM, at the cost of an increased complexity. In this paper, we focus our attention on the GSM scheme in which a fixed number of $0<N_{a}<N_{t}$ TX antennas are activated and a single IQ stream is transmitted, due to its promising tradeoff between achievable rate and implementation complexity. Compared with conventional spatial multiplexing (SMX) systems [13], [14], which typically need the same number of full TX chains (from baseband to RF) as the number of transmitted IQ streams, GSM offers three main advantages [2]: 1) reduced signal processing complexity and hardware cost due to the fact that it needs a single RF chain at the TX; 2) improved energy efficiency at the TX [15], since a single power amplifier is needed; and 3) avoidance of the inter-channel interference at the receiver (RX), which significantly reduces the complexity of the maximum likelihood (ML) demodulator [16].

Due to the encoding of information into IQ and spatial domains, it is not an easy task to derive a tractable and closed-form expression for the channel capacity of SM/GSM systems. In [10], [17], [18], the total channel capacity of SM is formulated as the sum of the capacities of IQ and spatial domains. The computation of the capacity of the spatial domain requires numerical integrations and no closedform expression is available. An analytical expression for the average ergodic channel capacity of GSM systems is introduced in [19]. In [20], [21], the authors propose a lowerbound for the mutual information of SM/GSM systems that assume Gaussian signaling for IQ symbols. An approximate capacity expression, in which the channel capacity in spatial domain is computed by using a multivariate Taylor series expansion, is presented in [22]. In [23], the authors derived a lower-bound of the mutual information of GSM in millimeterwave (mmWave) sparsely scattered channels. In [24], unlike the previous papers that assume Gaussian signaling for the IQ symbols, a lower-bound for the mutual information of SMmultiple input single output (MISO) systems that employ a discrete IQ modulation alphabet is derived. And the analysis is generalized for application to GSM systems in [25]. In [26], the authors introduced a lower-bound expression for the mutual information of SM with discrete IQ signaling in nonorthogonal multiple access systems.

To circumvent direct calculation of the channel capacity of SM/GSM systems, we propose to employ channel (or simply) cut-off rate for optimizing the performance of SM/GSM systems. To the best of our knowledge, this is the first time that cut-off rate is utilized for optimizing the performance of SM/GSM systems. Indeed, just a few available research works are focused on optimizing the cut-off rate of conventional MIMO. In [9], the authors consider SMX-MIMO and propose a phase precoder design that increases the averaged cut-off rate with respect to channel statistics. Precoding schemes that optimize the cut-off rate of MIMO systems, under imperfect channel estimation, are proposed in [27], [28]. In [29], [30], the authors propose new designs for the IQ modulation to optimize the cut-off rate of MIMO systems.

Against this background, the contributions made by this paper are as follows:

1) We propose to utilize cut-off rate as a performance metric for optimizing the mutual information (i.e., channel capacity) of the SM/GSM systems that employ a discrete IQ modulation alphabet. We show that the cut-off rate of SM/GSM systems can be formulated in a closed-form expression, which is easier to compute and to optimize compared with the actual channel capacity. Thus, cut-off rate is proved to be a convenient surrogate of channel capacity for system optimization and design.

2) We propose four different precoding schemes for increasing the cut-off rate of SM/GSM. The proposed precoding schemes employ different strategies for system optimization, which include grouping the TX antennas, applying singular value decomposition (SVD) or SVDdiscrete Fourier transform (DFT) pre-processing and then selecting a subset of inputs, and employing DFT precoding. We prove, also, that the precoding scheme based on SVD-DFT pre-processing usually outperforms its counterpart based on SVD pre-processing.

3) We evaluate the performance of the proposed precoding schemes with the aid of numerical simulations. All schemes, with the exception of DFT precoding, provide gains in low to moderate signal-to-noise ratio (SNR) regime. The DFT precoding scheme, on the other hand, is shown to outperform the other schemes in moderate to high SNR regime and especially in highly correlated channels.

4) We show that the improvement of the cut-off rate of SM/GSM systems that is obtained by using the proposed precoding schemes is comparable with the improvement of the mutual information that is computed numerically. Furthermore, we study channel capacity obtained by the proposed precoding schemes when discrete IQ signaling is replaced by Gaussian signaling. As a side product, we show that the proposed precoding schemes can be applied for enhancing the cut-off rate of SMX-MIMO systems as well. However, compared with SM/GSM systems, the gain is smaller. Meanwhile, we prove that optimizing symbol error probability (SEP) leads to smaller improvements of mutual information compared with those obtained by maximizing cut-off rate. Last but not least, we demonstrate that the proposed precoding schemes, though originally designed for SM/GSM, are also applicable to quadrature spatial modulation (QSM).

The rest of the paper is organized as follows. In Section II, we introduce the system model and derive the cut-off rate for SM/GSM systems. In Section III, we propose four precoding schemes that aim to increase cut-off rate. In Section IV, we illustrate simulation results of the cut-off rate for the proposed precoding schemes, and discuss the advantages and limitations of each of them. Finally, Section V concludes this paper.

Notation: Lowercase bold symbols denote column vectors; uppercase bold symbols denote matrices; |. | and ||$\cdot||$ denote 
absolute value and $\mathrm{L}_{2}$-norm, respectively; $\exp (\cdot), \log _{2}(\cdot)$, $\mathfrak{R}\{\cdot\}$ and $\mathbb{E}\{\cdot\}$ denote exponential function, base-two logarithm, the real part of a complex number and the mean (expected) value of a random variable, respectively; $\mathbf{j}$ denotes the imaginary unit; $p(\cdot), \mathcal{H}(\cdot)$ and $\mathcal{I}(\cdot)$ denote probability, entropy, and mutual information, respectively; $\mathbf{A}(i, j)$ denoted the $j$-th element of the $i$-th row of matrix $\mathbf{A} ; \mathbf{B}(:, j)$ denotes the $j$-th column of matrix $\mathbf{B} ; \mathcal{C N}(\cdot, \cdot)$ denotes a circularly symmetric complex Gaussian random variable.

\section{SySTEM MODEL}

The receive signal vector of a $N_{t} \times N_{r}$ SM/GSM system can be formulated as follows:

$$
\mathbf{y}=\frac{1}{\sqrt{N_{a}}} \mathbf{H e}_{i} s_{m}+\mathbf{n}
$$

where $\mathbf{H} \in \mathbb{C}^{N_{r} \times N_{t}}$ is the channel matrix and $s_{m}$ ( $m=$ $1, \ldots, M)$ is an IQ (e.g., $M$-QAM) symbol. The number of active TX antennas during each symbol interval is $N_{a}$. Hence, $N_{c}=\left(\begin{array}{l}N_{t} \\ N_{a}\end{array}\right)$ TX antenna activation patterns are used ${ }^{1}$, where each TX antenna activation pattern consists of a unique combination of $N_{a}$ active TX antennas. Spatial symbol $\mathbf{e}_{i}$ is a $N_{t} \times 1$ vector with $N_{a}$ ones (representing the active TX antennas) and $N_{t}-N_{a}$ zeros (representing the inactive TX antennas), and $i=1, \ldots, N_{c}$ is the spatial symbol index. Each spatial symbol specifies a unique TX antenna activation pattern. The indices of the active TX antennas for the $i$-th TX antenna activation pattern are denoted by a set $\Omega_{i}$. Finally, $\mathbf{n} \in \mathbb{C}^{N_{r} \times 1}$ is a noise vector whose distribution is $\mathcal{C N}\left(\mathbf{0}, N_{0} \mathbf{I}\right)$, where $N_{0}$ denotes the noise variance and $\mathbf{I}$ is a identity matrix. Since the data bits are encoded in both the IQ and spatial domains, the total number of GSM symbols is equal to $N_{\mathrm{GSM}}=N_{c} M$. As a result, $\log _{2} N_{\mathrm{GSM}}=\log _{2}\left(N_{c} M\right)$ bits per channel use (bpcu) are transmitted.

\section{A. Cut-off Rate}

Based on the definition in [4], the cut-off rate of GSM systems can be formulated, by assuming equiprobable GSM symbols, as follows:

$R_{0}=-\log _{2}\left[\frac{1}{N_{\mathrm{GSM}}^{2}} \int_{\mathbf{y}} \sum_{i, j, m, n} \sqrt{p\left(\mathbf{y} \mid s_{m}, \mathbf{e}_{i}\right) p\left(\mathbf{y} \mid s_{n}, \mathbf{e}_{j}\right)} \mathrm{d} \mathbf{y}\right]$

for $i, j=1, \ldots, N_{c}$ and $m, n=1, \ldots, M$. The conditional probability density function of the received data can be written as follows:

$$
p\left(\mathbf{y} \mid s_{m}, \mathbf{e}_{i}\right)=\frac{1}{\left(\pi N_{0}\right)^{N_{r}}} \exp \left(-\frac{\left\|\mathbf{y}-\mathbf{H} \mathbf{e}_{i} s_{m} / \sqrt{N_{a}}\right\|^{2}}{N_{0}}\right) .
$$

\footnotetext{
${ }^{1}$ We assume the use all the TX antenna activation patterns and do not restrict $N_{c}$ to be a power of 2 . This can be realized by using the methods proposed in [31], [32].
}

By inserting (3) in (2) and with the aid of some algebraic manipulations, the cut-off rate of GSM can be written as follows:

$$
R_{0}=-\log _{2}\left[\frac{1}{N_{\mathrm{GSM}}^{2}} \sum_{i, j, m, n} \exp \left(-\frac{\Phi_{m, n, i, j}}{4 N_{0}}\right)\right],
$$

where the Euclidean distance between two GSM symbols at the $\mathrm{RX}$ is equal to:

$$
\Phi_{m, n, i, j}=\frac{1}{N_{a}}\left\|\mathbf{H}\left(\mathbf{e}_{i} s_{m}-\mathbf{e}_{j} s_{n}\right)\right\|^{2} .
$$

It is worth mentioning that the cut-off rate in (2) is computed by assuming that the GSM symbols are equally probable. This assumption is made because this is a typical operating scenario of communication systems. Since the definition of cut-off rate in (4) requires channel state information at TX (CSIT), the performance may be further improved by optimizing the probability of emitting GSM symbols as a function of CSIT [4]. This is an interesting generalization of the study reported in the presented paper. Due to space limitations, however, it is postponed to future research. In the present paper, in fact, we are interested in focusing our attention to the sole impact of TX precoding.

\section{Proposed Precoding Schemes}

In this section, we introduce four precoding schemes that are capable of improving the cut-off rate of GSM systems ${ }^{2}$. The first precoding scheme is based on grouping together TX antennas that have the smallest Euclidean distance among them. The grouped TX antennas are activated together for data transmission. The second and third precoding schemes first apply SVD and SVD-DFT pre-processing, respectively, and then select a subset of active inputs ${ }^{3}$. The fourth precoding scheme is based on maximizing, for each TX antenna activation pattern, the minimum Euclidean distance between different GSM symbols at the RX.

\section{A. TX Antenna Grouping Precoding}

From a direct inspection of (4), we note that the cut-off rate depends on the Euclidean distances between different GSM symbols (5). More specifically, the smallest Euclidean distances constitute the dominant terms. Accordingly, the first precoding scheme is based on optimizing cut-off rate by grouping the TX antennas whose channel impulse responses have the smallest Euclidean distance. The grouped TX antennas are activated simultaneously, and, thus, they can be

\footnotetext{
${ }^{2}$ As conventional GSM works without the knowledge of CSIT, it may seem unfair to compare it with GSM that exploits CSIT. However, this is a common practice in the literature in order to understand the benefits of CSIT as well.

${ }^{3}$ Both SVD and SVD-DFT precoding schemes transmit signal from all $N_{t}$ TX antennas and therefore SM/GSM with SVD or SVD-DFT precoding schemes requires higher hardware complexity (i.e., $N_{t}$ RF chains at the TX) than conventional SM/GSM which needs only one TX RF chain. Utilizing more RF chains at the TX is a common practice in SM/GSM systems that exploit the knowledge of CSIT in order to achieve better system performances [33]. In addition, the operating principle of some SM schemes exploits CSIT, such as receive spatial modulation (RSM), and thus $N_{t} \mathrm{RF}$ chains are required at the TX [34], [35].
} 


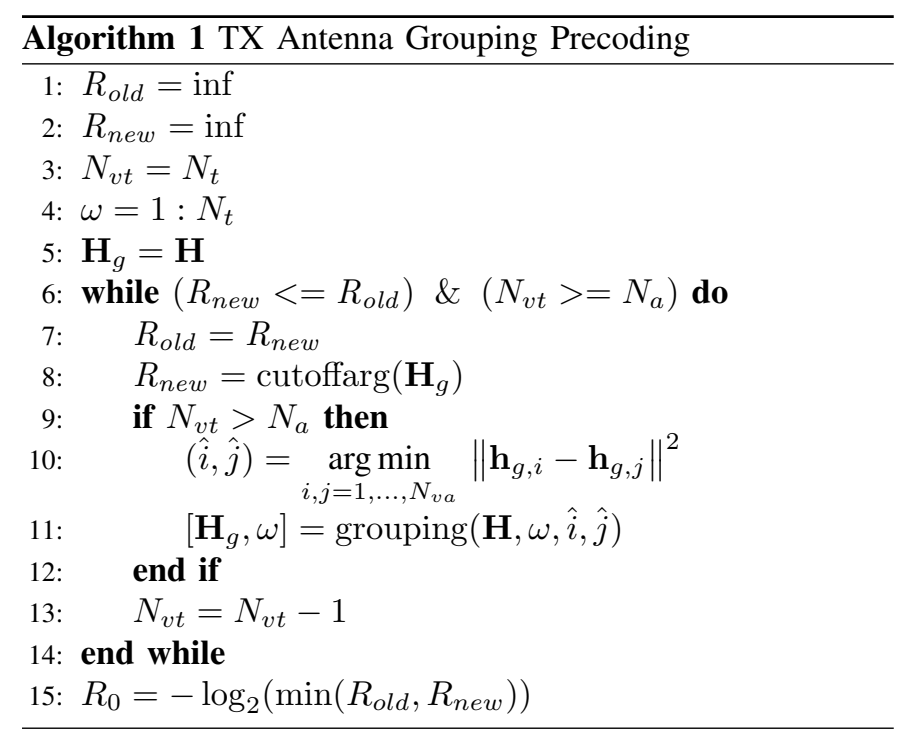

viewed as a virtual TX antenna whose impulse response is the normalized sum of the channel impulse responses of the physical TX antennas, i.e., H. Accordingly, the RX signal after applying the proposed TX antenna grouping algorithm can be formulated as follows:

$$
\mathbf{y}=\frac{1}{\sqrt{N_{a}}} \mathbf{H}_{g} \hat{\mathbf{e}}_{i} s_{m}+\mathbf{n},
$$

where $\mathbf{H}_{g} \in \mathbb{C}^{N_{r} \times N_{v t}}$ is the equivalent channel matrix after TX antenna grouping and $N_{v t}$ is the number of virtual TX antennas with $N_{v t} \leq N_{t}$. Spatial symbol $\hat{\mathbf{e}}_{i}$ is a $N_{v t} \times 1$ vector with $N_{a}$ ones (representing the active virtual TX antennas) and $N_{v t}-N_{a}$ zeros (representing the inactive virtual TX antennas), and $i=1, \ldots,\left(\begin{array}{c}N_{v t} \\ N_{a}\end{array}\right)$ is the index of the active spatial symbol.

In general, a virtual TX antenna is made of the scaled summation of one or more physical TX antennas. An example of TX antenna grouping is provided as follows.

Example 1. Consider a GSM system with $N_{t}=8$ physical TX antennas and let the grouping set be $\omega=$ $\{\{1,3\}, 2,\{4,5,7\},\{6,8\}\}$. The matrix $\mathbf{H}_{g}$ consists of $N_{v t}=$ 4 columns as follows: $\mathbf{h}_{g 1}=\left(\mathbf{h}_{1}+\mathbf{h}_{3}\right) / \sqrt{2}, \mathbf{h}_{g 2}=\mathbf{h}_{2}$, $\mathbf{h}_{g 3}=\left(\mathbf{h}_{4}+\mathbf{h}_{5}+\mathbf{h}_{7}\right) / \sqrt{3}$ and $\mathbf{h}_{g 4}=\left(\mathbf{h}_{6}+\mathbf{h}_{8}\right) / \sqrt{2}$. We assume that the transmit power is uniformly distributed among the TX antenna groups and equally allocated within each group. Hence, the sum is normalized by the number of antennas in order to keep the total TX power constant.

The precoding scheme based on TX antenna grouping is detailed in Algorithm 1. We note that the argument inside the logarithm function of (4) is minimized in order to maximize the cut-off rate $R_{0}$. As a starting point, $N_{v t}$ is set equal to $N_{t}$ and $\omega$ is initialized to the $N_{t}$ physical antennas. In the subsequent steps, the TX antennas are grouped iteratively and the argument of the logarithm function in (4) is obtained. These steps are repeated as long as the logarithm function is decreasing $\left(R_{\text {new }} \leq R_{\text {old }}\right)$ and the number of virtual TX antennas is greater than or equal to $N_{a}$. In particular, the argument of the logarithm function in (4) for a given channel matrix $\mathbf{H}_{g}$ is computed as follows (line 8 of Algorithm 1):

$R_{n e w}=\frac{1}{\left(\left(\begin{array}{c}N_{v t} \\ N_{a}\end{array}\right) M\right)^{2}} \sum_{i, j, m, n} \exp \left[-\frac{\left\|\mathbf{H}_{g}\left(\hat{\mathbf{e}}_{i} s_{m}-\hat{\mathbf{e}}_{j} s_{n}\right)\right\|^{2}}{4 N_{0} N_{a}}\right]$,

where $i, j=1, \ldots,\left(\begin{array}{c}N_{v t} \\ N_{a}\end{array}\right)$ and $m, n=1, \ldots, M$. Then, the indices of the virtual TX antennas that have the smallest Euclidean distance between their impulse responses are identified and grouped together. These steps are in lines 10 and 11 of Algorithm 1, which are executed only if $N_{v t}>N_{a}$ in order to avoid antenna groupings with $N_{v t}<N_{a}$. At each iteration of Algorithm 1, the number of virtual TX antennas $N_{v t}$ is decreased by one. Cut-off rate is computed ${ }^{4}$ in the last step.

\section{B. SVD-Based Precoding with Input Selection}

The second precoding scheme is based on SVD preprocessing at the TX. SVD precoding is often used in multistream SMX-MIMO systems, and, therefore, it constitutes an attractive solution for communication devices that incorporate SMX and GSM operation modes and that use them based on the required rate and performance. By applying SVD decomposition to the channel matrix $\mathbf{H}$, we obtain $\mathbf{H}=\mathbf{U} \boldsymbol{\Lambda} \mathbf{V}^{\mathrm{H}}$, where $\mathbf{U}$ and $\mathbf{V}$ are unitary matrices and $\boldsymbol{\Lambda}$ is a diagonal matrix whose singular values are, assuming $N_{t} \leq N_{r}$, $\lambda_{1}, \ldots, \lambda_{N_{t}}$. By using the matrix $\mathbf{V}$ as the precoding matrix, the RX signal vector can be written as follows:

$$
\begin{aligned}
\mathbf{y} & =\frac{1}{\sqrt{N_{a}}} \mathbf{H V \mathbf { e } _ { i }} s_{m}+\mathbf{n}=\frac{1}{\sqrt{N_{a}}} \mathbf{U} \boldsymbol{\Lambda} \mathbf{e}_{i} s_{m}+\mathbf{n} \\
& =\frac{1}{\sqrt{N_{a}}} \mathbf{G e}_{i} s_{m}+\mathbf{n} .
\end{aligned}
$$

The equivalent channel matrix (i.e., the product of channel and precoding matrices) is denoted by $\mathbf{G}$ and it is a column orthogonal matrix. More precisely, the transmission system in (7) consists of $N_{t}$ orthogonal sub-channels that correspond to the inputs of $\mathbf{G}$. If $N_{a}=1$, in particular, the Euclidean distance between two GSM symbols is as follows:

$$
\begin{aligned}
\Phi_{m, n, i, j} & =\left\|\mathbf{G}\left(\mathbf{e}_{i} s_{m}-\mathbf{e}_{j} s_{n}\right)\right\|^{2}=\left\|\mathbf{g}_{i} s_{m}-\mathbf{g}_{j} s_{n}\right\|^{2} \\
& =\left\|\mathbf{g}_{i} s_{m}\right\|^{2}+\left\|\mathbf{g}_{j} s_{n}\right\|^{2}=\lambda_{i}^{2}\left|s_{m}\right|^{2}+\lambda_{j}^{2}\left|s_{n}\right|^{2},
\end{aligned}
$$

where $\mathbf{g}_{i}$ and $\mathbf{g}_{j}$ are the $i$-th and $j$-th column of $\mathbf{G}$. Equation (8) is useful for understanding why the grouping strategy employed in Section III-A is not effective in this case. Assume, e.g., that two arbitrary columns, $N_{1}$ and $N_{2}$ with $1 \leq N_{1}<$ $N_{2} \leq N_{t}$, of $\mathbf{G}$ are grouped together. The resulting impulse response would be equal to $\left(\mathbf{g}_{N_{1}}+\mathbf{g}_{N_{2}}\right) / \sqrt{2}$ and the following would hold:

$$
\frac{\left\|\mathbf{g}_{N_{1}}+\mathbf{g}_{N_{2}}\right\|^{2}}{2}=\frac{\lambda_{N_{1}}^{2}+\lambda_{N_{2}}^{2}}{2} \leq \lambda_{N_{1}}^{2}=\left\|\mathbf{g}_{N_{1}}\right\|^{2} .
$$

In other words, the power gain without grouping is larger than the power gain with grouping. From a direct inspection of (8) and (9), we note, in addition, that by grouping the columns of

\footnotetext{
${ }^{4}$ It should be noticed that $R_{\text {new }}$ determines the cut-off rate $R_{0}$ only when $R_{\text {new }}<R_{\text {old }}$ and $R_{\text {new }}$ is computed when $N_{v t}=N_{a}$. In all other situations $R_{\text {old }}$ determines $R_{0}$.
} 


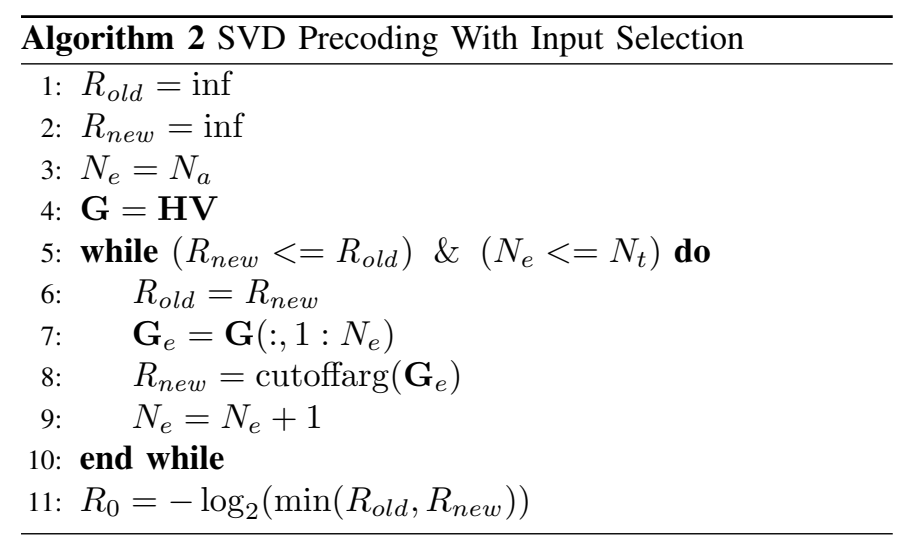

G we obtain an Euclidean distance between two GSM symbols that is smaller than that corresponding to the larger singular value of G. Based on this example, we evince that antenna grouping is not a suitable approach based on (7). A better strategy is, in fact, to select only a subset of active columns of $\mathbf{G}$ rather than grouping them. The column orthogonality property of $\mathbf{G}$ facilitates this task. Based on (9), in fact, the columns with the largest norm can be selected to optimize cut-off rate. This implies that cut-off rate can be maximized by selecting the largest singular values of the channel matrix H. By assuming, without loss of generality, that the singular values are sorted in descending order, the first $N_{e}\left(N_{a} \leq\right.$ $N_{e} \leq N_{t}$ ) columns of $\mathbf{G}$ need to be selected. Under these assumptions, the RX signal vector in (7) can be re-written as:

$$
\mathbf{y}=\frac{1}{\sqrt{N_{a}}} \mathbf{G}_{e} \overline{\mathbf{e}}_{i} s_{m}+\mathbf{n},
$$

where $\mathbf{G}_{e} \in \mathbb{C}^{N_{r} \times N_{e}}$ consists of the first $N_{e}$ columns of G and $\overline{\mathbf{e}}_{i}$ is a spatial symbol, i.e., $N_{e} \times 1$ vector with $N_{a}$ ones (representing the active columns of $\mathbf{G}_{e}$ ) and $N_{e}-N_{a}$ zeros (representing the inactive column of $\mathbf{G}_{e}$ ), where $i=$ $1, \ldots,\left(\begin{array}{l}N_{e} \\ N_{a}\end{array}\right)$ is the index of the active spatial symbol.

The details of the proposed precoding scheme are reported in Algorithm 2. Similar to Algorithm 1, we aim to minimize the argument of the logarithm function of the cut-off rate. The difference between Algorithm 2 and Algorithm 1 is that, at each iteration, the subset of active columns of $\mathbf{G}$ is increased rather than decreased. The initialization of Algorithm 2 is similar to that of Algorithm 1. In particular, $N_{e}$ is set equal to $N_{a}$ because we have to activate at least $N_{a}$ columns of G. In the next few steps, we iteratively increase the set of active columns of $\mathbf{G}$ until the argument of the logarithm function of the cut-off rate decreases. The argument of the logarithm function of cut-off rate is calculated in line 8 of Algorithm 2 as follows:

$R_{\text {new }}=\frac{1}{\left(\left(\begin{array}{c}N_{e} \\ N_{a}\end{array}\right) M\right)^{2}} \sum_{i, j, m, n} \exp \left[-\frac{\left\|\mathbf{G}_{e}\left(\overline{\mathbf{e}}_{i} s_{m}-\overline{\mathbf{e}}_{j} s_{n}\right)\right\|^{2}}{4 N_{0} N_{a}}\right]$,

where $i, j=1, \ldots,\left(\begin{array}{l}N_{e} \\ N_{a}\end{array}\right)$ and $m, n=1, \ldots, M$. It is worth noting that the computational complexity of this step can be significantly reduced. At each iteration, in fact, we need to calculate only the Euclidean distances that are determined

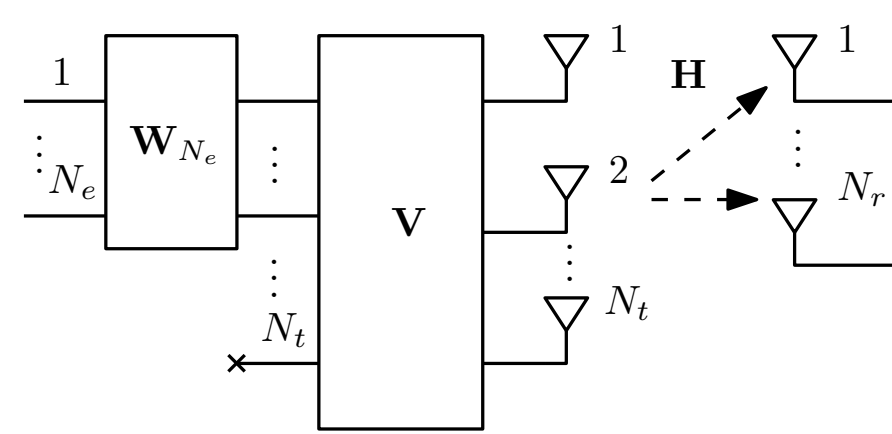

Fig. 1. Block diagram of GSM with SVD-DFT precoding with the DFT matrix $\mathbf{W}_{N_{e}}$ and the matrix $\mathbf{V}$ from the SVD of the channel matrix $\mathbf{H}$. By removing the precoding matrix $\mathbf{W}_{N_{e}}$ we get a block diagram of GSM with SVD precoding.

by the $N_{e}$-th column of $\mathbf{G}$ because all the other Euclidean distances have been pre-computed at the previous iterations.

\section{SVD-DFT Precoding With Input Selection}

Based on the previous section, we evince that SVD precoding when applied to GSM systems, similar to SMX systems [14], transforms the channel matrix into a set of orthogonal sub-channels. From [14], [36], it is known that SMX systems that employ SVD precoding achieve the largest channel capacity if the water-filling power allocation strategy is utilized, i.e., the higher power is allocated to the subchannels that correspond to the larger singular values of the channel matrix. In [37], the authors have proposed a precoding scheme aiming to improve the error performance of SMX systems, which transforms a SMX system that consists of orthogonal sub-channels with unequal allocated power into a SMX system that consists of non-orthogonal sub-channels with equal power allocation. This is obtained by applying a DFT precoding matrix. In this paper, we are not interested in power allocation, as this is left to future research work. We, however, introduce and optimize, from the cut-off rate point of view, a precoding scheme that takes inspiration from the SVDDFT precoding introduced in [37]. The proposed precoding scheme is depicted in Fig. 1. The main difference between the SVD-DFT precoding scheme in Fig. 1 and the precoding scheme in [37] is the size of DFT precoding matrix. In [37], the authors have used a DFT precoding matrix with fixed size equal to $N_{t} \times N_{t}$. In Fig. 1, on the other hand, we use a DFT precoding matrix whose size is variable and is equal to $N_{e} \times N_{e}$, where $N_{e}$ is the number of active inputs that is not fixed but changes similar to the SVD precoding matrix of the previous section. Another difference compared with [37] lies in considering a GSM system and, thus, transmitting a single IQ stream but encoding some information bits into the columns of the TX matrix. In the following, we first introduce the SVDDFT precoding scheme for application to GSM systems and then we compare the minimum Euclidean distances of SVD and SVD-DFT precoding schemes.

1) SVD-DFT Precoding: Based on Fig. 1, the RX signal of a GSM system with SVD-DFT precoding can be formulated 
as follows:

$$
\mathbf{y}=\frac{1}{\sqrt{N_{a}}} \mathbf{G}_{e} \mathbf{W}_{N_{e}} \overline{\mathbf{e}}_{i} s_{m}+\mathbf{n}=\frac{1}{\sqrt{N_{a}}} \mathbf{F} \overline{\mathbf{e}}_{i} s_{m}+\mathbf{n} .
$$

which is obtained from (7) by multiplying the precoding matrix $\mathbf{V}$ by the DFT matrix whose elements are the following:

$$
\mathbf{W}_{N_{e}}(i, j)=\frac{1}{\sqrt{N_{e}}} \exp \left(-\mathbf{j} \frac{2 \pi(i-1)(j-1)}{N_{e}}\right),
$$

and the matrix $\mathbf{F}$ is defined as follows:

$$
\mathbf{F}=\mathbf{G}_{e} \mathbf{W}_{N_{e}}=\mathbf{G}\left(:, 1: N_{e}\right) \mathbf{W}_{N_{e}} .
$$

The scaling factor $1 / \sqrt{N_{e}}$ ensures that the average signal power remains constant, i.e., $\operatorname{Tr}\left(\mathbf{W}_{N_{e}} \mathbf{W}_{N_{e}}^{\mathrm{H}}\right)=N_{e}$. The algorithm for maximizing cut-off rate is the same as Algorithm 2. The only difference is that, at each iteration of the algorithm, the matrix $\mathbf{G}_{e}$ is replaced by the matrix $\mathbf{F}$.

2) Performance Comparison of SVD Precoding and SVDDFT Precoding: In order to get insight into the achievable cut-off rate of SVD and SVD-DFT precoding schemes, we study and compare the minimum Euclidean distance of the GSM symbols at the RX when the two precoding schemes are used. Since in GSM systems the data is encoded into both IQ and spatial domains, computation of the minimum Euclidean distance is more challenging than for SMX systems [37]. To make the mathematical development tractable, therefore, we restrict our analysis to SM systems, i.e., we assume $N_{a}=1$. The main result is reported in Theorem 2. However, we first introduce some intermediate results that are useful for the proof of the main theorem. It is worth mentioning that, with the exception of Lemma 2 that is similar to [37, Lemma 2], all the results in the following proof are new and, to the best of our knowledge, have never been published.

Theorem 1. For a SM system with SVD precoding and $M$ QAM modulation $(M \geq 4)$, the minimum Euclidean distance between $R X$-side SM symbols is:

$$
\Phi_{\min }=2 \lambda_{N_{e}}^{2} \mathrm{r}_{\min }^{2},
$$

where $\mathrm{r}_{\min }=\min _{m}\left|s_{m}\right|$.

Proof: If two SM symbols differ by the transmitted IQ symbol but activate the same TX antenna $(m \neq n, i=j)$, the Euclidean distance between them is:

$$
\begin{aligned}
\Phi_{m, n, i, j} & =\left\|\mathbf{G}_{e}(:, i)\left(s_{m}-s_{n}\right)\right\|^{2}=\left\|\mathbf{G}_{e}(:, i)\right\|^{2}\left|s_{m}-s_{n}\right|^{2} \\
& \geq \lambda_{N_{e}}^{2} \mathrm{~d}_{\min }^{2}
\end{aligned}
$$

where $\mathrm{d}_{\text {min }}=\min _{m, n(m \neq n)}\left|s_{m}-s_{n}\right|$. If two SM symbols differ by the active TX antenna $(i \neq j)$, the Euclidean distance between them is:

$$
\begin{aligned}
\Phi_{m, n, i, j} & =\left\|\mathbf{G}_{e}(:, i) s_{m}-\mathbf{G}_{e}(:, j) s_{n}\right\|^{2} \\
& =\left\|\mathbf{G}_{e}(:, i) s_{m}\right\|^{2}+\left\|\mathbf{G}_{e}(:, j) s_{n}\right\|^{2} \\
& =\left|\lambda_{i}\right|^{2}\left|s_{m}\right|^{2}+\left|\lambda_{j}\right|^{2}\left|s_{n}\right|^{2} \\
& \geq\left(\lambda_{N_{e}-1}^{2}+\lambda_{N_{e}}^{2}\right) \mathrm{r}_{\min }^{2} .
\end{aligned}
$$

where the last inequality holds for $m \neq n$ and $m=n$, provided that $\left|s_{m}\right|=\left|s_{n}\right|=\mathrm{r}_{\min }$. Since $\mathrm{d}_{\min }^{2}=2 \mathrm{r}_{\min }^{2}$ for $M$-QAM constellations if $M \geq 4$ and the inequality $\lambda_{N_{e}-1}^{2}+\lambda_{N_{e}}^{2} \geq 2 \lambda_{N_{e}}^{2}$ holds, the minimum Euclidean distance between RX-side SM symbols is given by (14).

Lemma 1. The inner-product of the $i$-th and $j$-th columns of matrix $\mathbf{F}$ is given by:

$$
\left\langle\mathbf{f}_{i}, \mathbf{f}_{j}\right\rangle=\frac{1}{N_{e}} \sum_{m=1}^{N_{e}} \lambda_{m}^{2} \exp \left(\mathbf{j} \frac{2 \pi(m-1)(i-j)}{N_{e}}\right) .
$$

Proof: The $i$-th column of $\mathbf{F}$ is $\mathbf{f}_{i}=\mathbf{G}_{e} \mathbf{W}_{N_{e}}(:, i)$. So, we obtain:

$$
\begin{aligned}
\left\langle\mathbf{f}_{i}, \mathbf{f}_{j}\right\rangle & =\mathbf{f}_{i}^{\mathrm{H}} \mathbf{f}_{j}=\mathbf{W}_{N_{e}}^{\mathrm{H}}(:, i) \mathbf{G}_{\mathrm{e}}^{\mathrm{H}} \mathbf{G}_{\mathrm{e}} \mathbf{W}_{N_{e}}(:, j) \\
& =\frac{1}{N_{e}} \sum_{m=1}^{N_{e}} \lambda_{m}^{2} e^{\mathbf{j} \frac{2 \pi(m-1)(i-1)}{N_{e}}} e^{-\mathbf{j} \frac{2 \pi(m-1)(j-1)}{N_{e}}} \\
& =\frac{1}{N_{e}} \sum_{m=1}^{N_{e}} \lambda_{m}^{2} e^{\mathbf{j} \frac{2 \pi(m-1)(i-j)}{N_{e}}}
\end{aligned}
$$

from which the proof follows.

Lemma 2. All columns of matrix $\mathbf{F}$ have the same norm, which is equal to:

$$
\left\|\mathbf{f}_{i}\right\|^{2}=\frac{1}{N_{e}} \sum_{m=1}^{N_{e}} \lambda_{m}^{2}, \quad i=1, \ldots, N_{e} .
$$

Proof: If follows directly from Lemma 1.

Lemma 3. Let $N>1$ be a positive integer and $k \in$ $\{ \pm 1, \pm 2, \ldots, \pm(N-1)\}$. The following equation holds true:

$$
\sum_{m=1}^{N} \cos \left(\frac{2 \pi(m-1) k}{N}\right)=0 .
$$

Proof: It is known that, except for the first row and column, the sum of the elements of each row and column of a DFT matrix is equal to zero. Therefore, we have

$$
\sum_{m=1}^{N} \exp \left(\mathbf{j} \frac{2 \pi(m-1) k}{N}\right)=0
$$

where $k$ takes any value in set $\{-1,-2, \cdots,-(N-1)\}$. Accordingly, the real part of (20) must be equal to zero and (19) holds true for $k<0$. Since the cosine is an even function, (19) is valid for $k>0$ as well.

Theorem 2. For a SVD-DFT precoding assisted SM systems that employ M-QAM modulation in the IQ domain, and have parameters $M \geq 4$ and $N_{e}>1$, the minimum Euclidean distance is greater than the $\Phi_{\min }$ given in (14).

Proof: Similar to Theorem 1, we define $\mathrm{d}_{\min }=$ $\min _{m, n(m \neq n)}\left|s_{m}-s_{n}\right|$ and $\mathrm{r}_{\min }=\min _{m}\left|s_{m}\right|$. Three cases are analyzed separately. 
Case 1. Assume that two SM symbols have different IQ constellation points but the activated TX antenna is the same. The minimum Euclidean distance between the corresponding two RX-side SM symbols is given by

$$
\begin{aligned}
\Phi_{m, n, i, j} & =\left\|\mathbf{f}_{i}\left(s_{m}-s_{n}\right)\right\|^{2}=\left\|\mathbf{f}_{i}\right\|^{2}\left|s_{m}-s_{n}\right|^{2} \\
& \geq \frac{\sum_{k=1}^{N_{e}} \lambda_{k}^{2}}{N_{e}} \mathrm{~d}_{\min }^{2}=2 \frac{\sum_{k=1}^{N_{e}} \lambda_{m}^{2}}{N_{e}} \mathrm{r}_{\min }^{2}
\end{aligned}
$$

From (14) and taking into account that:

$$
2 \frac{\sum_{k=1}^{N_{e}} \lambda_{k}^{2}}{N_{e}} \mathrm{r}_{\min }^{2} \geq 2 \lambda_{N_{e}}^{2} \mathrm{r}_{\min }^{2}
$$

we conclude that the minimum Euclidean distance of SM systems with SVD-DFT precoding is greater than its counterpart with SVD precoding.

Case 2. Assume that two SM symbols activate different TX antennas, but transmit the same IQ symbol. The minimum Euclidean distance between the corresponding two RX-side SM symbols is given by

$$
\begin{aligned}
\Phi_{m, n, i, j} & =\left\|\left(\mathbf{f}_{i}-\mathbf{f}_{j}\right) s_{n}\right\|^{2} \\
& =\left[\left\|\mathbf{f}_{i}\right\|^{2}+\left\|\mathbf{f}_{j}\right\|^{2}-2 \Re\left\{\mathbf{f}_{i}^{\mathrm{H}} \mathbf{f}_{j}\right\}\right]\left|s_{n}\right|^{2} \\
& \geq 2 \frac{\mathrm{r}_{\min }^{2}}{N_{e}} \sum_{k=1}^{N_{e}} \lambda_{k}^{2}\left[1-\cos \left(\frac{2 \pi(k-1)(i-j)}{N_{e}}\right)\right] .
\end{aligned}
$$

In (23), $i-j$ can take all possible values lying in the interval $\left[-N_{e}+1, N_{e}-1\right]$ except the zero value. In general, it is not easy to determine the value of $i-j$ that yields the minimum of (23). Therefore, we need to prove that (23) is greater than the minimum Euclidean distance from (14), i.e., $2 \lambda_{N_{e}}^{2} \mathrm{r}_{\min }^{2}$, for all possible values of $i-j$. In other words, we need to prove the following inequality:

$$
\frac{1}{N_{e}} \sum_{k=1}^{N_{e}} \lambda_{k}^{2}\left[1-\cos \left(\frac{2 \pi(k-1)(i-j)}{N_{e}}\right)\right] \geq \lambda_{N_{e}}^{2} .
$$

With the aid of Lemma 3, we obtain:

$$
N_{e} \lambda_{N_{e}}^{2}=\sum_{k=1}^{N_{e}} \lambda_{N_{e}}^{2}\left[1-\cos \left(\frac{2 \pi(k-1)(i-j)}{N_{e}}\right)\right] .
$$

By plugging (25) in (24), we obtain:

$$
2 \sum_{k=1}^{N_{e}}\left(\lambda_{k}^{2}-\lambda_{N_{e}}^{2}\right) \sin ^{2}\left(\frac{\pi(k-1)(i-j)}{N_{e}}\right) \geq 0 .
$$

The inequality in (26) implies that SVD-DFT precoding provides a larger minimum Euclidean distance than SVD precoding.

Case 3. Assume that two SM symbols have different IQ constellation points and that the activated TX antennas are different as well. The minimum Euclidean distance between the corresponding two SM symbols at the RX is the following

$$
\begin{aligned}
\Phi_{m, n, i, j}= & \left\|\mathbf{f}_{i} s_{m}-\mathbf{f}_{j} s_{n}\right\|^{2} \\
= & \left\|\mathbf{f}_{i}\right\|^{2}\left|s_{m}\right|^{2}+\left\|\mathbf{f}_{j}\right\|^{2}\left|s_{n}\right|^{2}-2 \mathfrak{R}\left\{\mathbf{f}_{i}^{\mathrm{H}} \mathbf{f}_{j} s_{m}^{*} s_{n}\right\} \\
= & \left\|\mathbf{f}_{i}\right\|^{2} r_{m}^{2}+\left\|\mathbf{f}_{j}\right\|^{2} r_{n}^{2}-2 r_{m} r_{n} \mathfrak{R}\left\{\mathbf{f}_{i}^{\mathrm{H}} \mathbf{f}_{j} \exp \left(\mathbf{j} \varphi_{m n}\right)\right\} \\
= & \frac{1}{N_{e}} \sum_{k=1}^{N_{e}} \lambda_{k}^{2}\left[r_{m}^{2}+r_{n}^{2}\right. \\
& \left.\quad-2 r_{m} r_{n} \cos \left(\varphi_{m n}+\frac{2 \pi(k-1)(i-j)}{N_{e}}\right)\right]
\end{aligned}
$$

where $r_{m}=\left|s_{m}\right|, r_{n}=\left|s_{n}\right|$ and $s_{m}^{*} s_{n}=r_{m} r_{n} \exp \left(\mathbf{j} \varphi_{m n}\right)$. In this case, the comparison of the minimum Euclidean distances is intractable. Thus, we restrict the analysis to the case study $r_{m}=r_{n}=r_{\min }$, which ensures to consider the minimum of the first two terms in (27). By applying a procedure similar to that utilized for computing (23)-(25), we obtain:

$$
2 \sum_{k=1}^{N_{e}}\left(\lambda_{k}^{2}-\lambda_{N_{e}}^{2}\right) \sin ^{2}\left(\frac{\varphi_{m n}}{2}+\frac{\pi(k-1)(i-j)}{N_{e}}\right) \geq 0
$$

which implies that, at least for $r_{m}=r_{n}=r_{\text {min }}$, SVD-DFT precoding is capable of increasing the minimum Euclidean distance compared to SVD precoding.

By comparing (22), (26) and (28), we evince that SVDDFT precoding is not capable of increasing the minimum Euclidean distance if $\lambda_{1}=\lambda_{2}=\ldots=\lambda_{N_{e}}$ holds true. This scenario, however, is unlikely to occur. In the worst case that it occurs, the precoding schemes would offer the same minimum Euclidean distance. Therefore, we conclude that SM systems with SVD-DFT precoding are expected to have a larger minimum Euclidean distance between different RX symbols, which, in turns, results into a better cut-off rate compared with SM systems that employ SVD precoding. The numerical results in Section V confirm this finding.

\section{DFT Precoding}

In this section, we consider a precoding scheme that aims to improve the cut-off rate of SM/GSM systems by selecting, for each possible TX antenna pattern, the precoding vector that provides the largest minimum Euclidean distance between RXside SM/GSM symbols [38]. The precoding vector is selected among a given set of candidate precoding vectors. Identifying this set is, however, a difficult and open issue. In the present paper, the set of candidate precoding vectors is obtained from the columns of the DFT matrix introduced in the previous section. For this reason, the scheme proposed in this section is referred to as DFT precoding. The GSM signal vector at the RX can be formulated as follows:

$$
\mathbf{y}=\frac{1}{\sqrt{N_{a}}} \mathbf{H} \tilde{\mathbf{P}} \tilde{\mathbf{e}}_{i} s_{m}+\mathbf{n},
$$

where $\tilde{\mathbf{P}} \in \mathbb{C}^{N_{t} \times N_{c}}$ is the precoding matrix and each column of $\tilde{\mathbf{P}}$ contains $N_{a}$ non-zero elements and $N_{t}-N_{a}$ zero elements. The positions of the non-zero elements correspond 


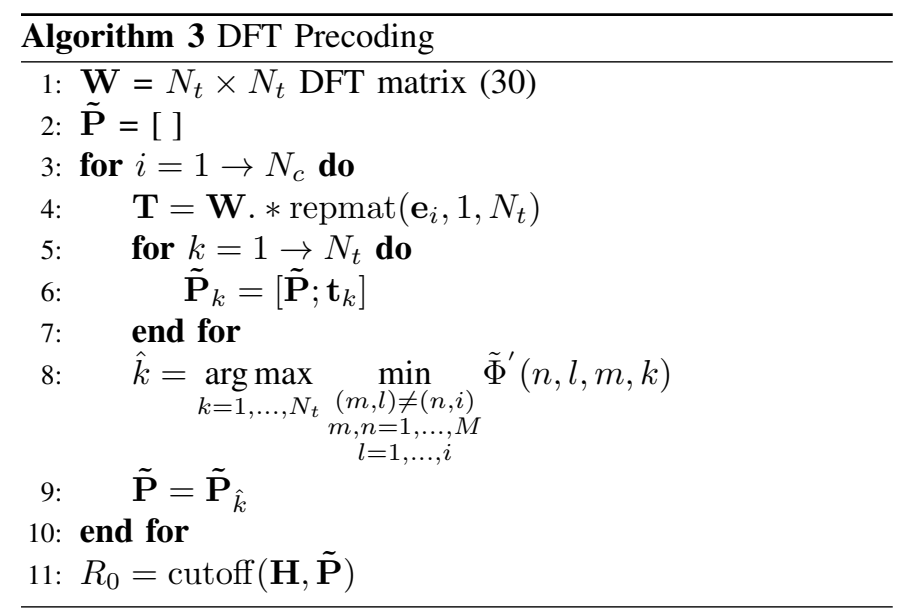

to the indices of the active TX antennas of the $i$-th TX antenna activation pattern. $\tilde{\mathbf{e}}_{i}$ is a spatial symbol, i.e., a $N_{c} \times 1$ vector with a single one and $N_{c}-1$ zero elements, and $i=1, \ldots, N_{c}$ is the spatial symbol index that specifies the activated TX antenna pattern.

The details of the proposed DFT precoding scheme for GSM systems are reported in Algorithm 3. First, we generate an $N_{t} \times N_{t}$ DFT matrix $\mathbf{W}$ whose elements are

$$
\mathbf{W}(i, j)=\exp \left(-\mathbf{j} \frac{2 \pi(i-1)(j-1)}{N_{t}}\right)
$$

Then, from $\mathbf{W}$, we construct the precoding candidate matrix $\mathbf{T} \in \mathbb{C}^{N_{t} \times N_{t}}$, which contains the set of candidate precoding vectors for a single TX antenna pattern. As far as the $i$-th $\left(i=1, \ldots, N_{c}\right)$ TX antenna activation pattern is concerned, the matrix $\mathbf{T}$ is constructed by taking the rows of $\mathbf{W}$ whose indices are determined by the elements of $\Omega_{i}$, i.e., the indices of the active TX antennas (see Section II). All the other rows of $\mathbf{T}$, whose indices are different from the elements of $\Omega_{i}$, contain only zero values. Therefore, each column $\mathbf{t}_{k}\left(k=1, \ldots, N_{t}\right)$ of $\mathbf{T}$ provides the candidate precoding vectors and has $N_{a}$ nonzero elements at the positions that correspond to the indices of the active TX antennas of the $i$-th TX antenna pattern. Subsequently, we construct $N_{t} \tilde{\mathbf{P}}_{k}$ matrices $\left(k=1, \ldots, N_{t}\right)$. Each $\tilde{\mathbf{P}}_{k}$ matrix is obtained by adding the $k$-th column $(k=$ $1, \ldots, N_{t}$ ) of $\mathbf{T}$ to the matrix $\tilde{\mathbf{P}}$ that is obtained at the previous iteration. Finally, we select, among all the $\tilde{\mathbf{P}}_{k}$ matrices, the matrix with the largest minimum Euclidean distance. Due to the constant increase of the number of columns of $\tilde{\mathbf{P}}$, the Euclidean distances are calculated as follows:

$$
\tilde{\Phi}^{\prime}(n, l, m, k)=\left\|\mathbf{H}\left(\tilde{\mathbf{P}}_{k}(:, i) s_{n}-\tilde{\mathbf{P}}_{k}(:, l) s_{m}\right)\right\|^{2} .
$$

Thus, at each iteration we need to calculate only the Euclidean distances that are affected by the candidate precoding vector $\mathbf{t}_{k}$. This procedure is repeated for each TX antenna activation pattern. After determining the precoding matrix $\tilde{\mathbf{P}}$, the cut-off rate is calculated as follows:

$$
\begin{gathered}
R_{0}=2 \log _{2}\left(M N_{c}\right)- \\
\log _{2}\left[\sum_{i, j, m, n} \exp \left(-\frac{\left\|\mathbf{H} \tilde{\mathbf{P}}\left(\tilde{\mathbf{e}}_{i} s_{m}-\tilde{\mathbf{e}}_{j} s_{n}\right)\right\|^{2}}{4 N_{0} N_{a}}\right)\right],
\end{gathered}
$$

where $i, j=1, \ldots, N_{c}$ and $m, n=1, \ldots, M$. In contrast to the other proposed precoding schemes, DFT precoding keeps the same number of GSM symbols and data rate as the original GSM system without precoding. Algorithm 3 provides the appropriate precoding vector for each TX antenna activation pattern and, therefore, it does not reduce the number of GSM symbols as the other precoding schemes. The consequences of this property are analyzed in Section IV.

\section{Simulation Results}

In this section, we evaluate the cut-off rate of the proposed precoding schemes with the aid of Monte Carlo simulations. The study is conducted for a typical indoor propagation channel. As a benchmark to quantify the gains of the proposed precoding schemes, a GSM system without precoding is considered [10], [12]. Besides cut-off rate, we study the impact of the precoding schemes on the mutual information of GSM systems, and show that an improvement of cut-off rate corresponds to an improvement of mutual information. Additionally, we evaluate capacity gains provided by the proposed precoding schemes, if discrete IQ signaling is replaced with Gaussian signaling. Further, we study suitability of the proposed precoding schemes for application to SMX systems and demonstrate that optimizing SEP, instead of cut-off rate, usually results in a smaller improvement of mutual information. Finally, we demonstrate that the proposed precoding schemes are also applicable to QSM.

\section{A. Cut-off Rate of SM/GSM}

At low-GHz frequencies (i.e., below $6 \mathrm{GHz}$ ), indoor channel are usually modeled by using the Rician distribution [39] and the channel matrix can be written as follows:

$$
\mathbf{H}=\sqrt{\frac{K}{K+1}} \mathbf{H}_{\mathrm{LOS}}+\sqrt{\frac{1}{K+1}} \mathbf{H}_{\mathrm{NLOS}},
$$

where $\mathbf{H}_{\mathrm{LOS}}$ and $\mathbf{H}_{\mathrm{NLOS}}$ are the line-of-sight (LOS) and non-LOS (NLOS) channel matrices, respectively. The element of $\mathbf{H}_{\mathrm{LOS}}$ that corresponds to the normalized channel coefficient between the $j$-th TX antenna and the $i$-th RX antenna is given by the Friis formula:

$$
\mathbf{H}_{\mathrm{LOS}}(i, j) \approx \frac{D}{d_{i j}} \exp \left(-\mathbf{j} \frac{2 \pi d_{i j}}{\lambda}\right),
$$

where $\lambda$ is the wavelength, $d_{i j}$ is the length of the LOS path between the antennas and $D$ is the distance between the midpoints of the TX and RX antenna uniform linear array (ULA). The inter-antenna separations of the TX and RX ULAs are assumed to be $\lambda / 2$, so that the inter-antenna interference can be assumed to be negligible. The elements of $\mathbf{H}_{\mathrm{NLOS}}$ are distributed according to $\mathcal{C N}(0,1)$. As Rician fading implies a scaled sum of LOS and NLOS channel components, we 
analyze the cut-off rate of the proposed precoding schemes for LOS and NLOS channel components separately. Hence, the Rician factor $K$ takes two values in our simulations: 0 and $+\infty$, which implies that either pure LOS channels or pure independent and identically distributed (i.i.d.) Rayleigh fading channels (i.e., NLOS channels) are considered. Using these two extreme channel models, we are able to shed light on the impact of the proposed precoding schemes on LOS and NLOS channel components, and obtain a more comprehensive overview of cut-off rate gains.

A MIMO system with $8 \mathrm{TX}$ and $8 \mathrm{RX}$ antennas is considered. The operating frequency is $f=5 \mathrm{GHz}$, i.e. $\lambda=6 \mathrm{~cm}$, and the inter-antenna separations of TX and RX ULAs, $d_{t}$ and $d_{r}$, are both $3 \mathrm{~cm}$. The distance between the TX and RX ULA is $D=10 \mathrm{~m}$. For a fair comparison among all the considered systems, we select parameters $N_{a}$ and $M$ such that the same data rate of $8 \mathrm{bpcu}$ is achieved.

1) Cut-Off Rate in i.i.d. Rayleigh Fading Channels: The cut-off rate of the different precoding schemes and the benchmark GSM system are compared against each other in Fig. 2 by assuming an i.i.d. Rayleigh fading channel model. We observe that the SVD-DFT precoding scheme provides the best cut-off rate at low to medium SNR. The SVD precoding scheme provides a slightly lower cut-off rate in the same SNR regime. In Fig. 2(b), for example, the SVD-DFT and SVD precoding schemes provide an improvement of the cutoff rate of about $1 \mathrm{bpcu}$ for $\mathrm{SNR}=-4 \mathrm{~dB}$ compared with the benchmark without precoding. On the other hand, SVD precoding does not offer good performance in medium to high SNR regime. The reason of this performance trend originates from the fact that the total number of GSM symbols for SVD precoding is constrained by its properties. The minimum Euclidean distance between different RX-side SM/GSM symbols, $\Phi_{\text {min }}$, for GSM with SVD precoding depends on primarily of the smallest singular value $\lambda_{N e}$ (14). With increasing the number of selected inputs of $\mathbf{G}, \lambda_{N_{e}}$ and $\Phi_{\min }$ are getting smaller. Therefore, SVD precoding, in medium to high SNR range, may not select the inputs (i.e., columns) of $\mathbf{G}$ that provide the smallest $\Phi_{\min }$ among RX-side GSM symbols, and total number of GSM symbols for SVD precoding may be smaller than its counterparts that adopt other precoding schemes. At the same time, the number of used GSM symbols becomes a very significant factor for cut-off rate as SNR increases. Due to these two effects, SVD precoding offers a worse cut-off rate, even compared to conventional GSM, in medium to high SNR regime. In contrast to SVD and SVDDFT precoding, the precoding scheme based on TX antenna grouping offers only a negligible cut-off rate improvement in low to medium SNR regime, which is justified in Appendix A.

In general, TX antenna grouping, SVD and SVD-DFT precoding transmit the TX signal from more than $N_{a}$ physical antennas per symbol interval, with the aim of improving cutoff rate by realizing a large array gain, which is especially important in the low SNR regime [10], [18]. Hence, in very low SNR regime it may occur that SVD and SVD-DFT precoding schemes select only $N_{a}$ active inputs, and the TX antenna grouping scheme has only $N_{a}$ virtual TX antennas.

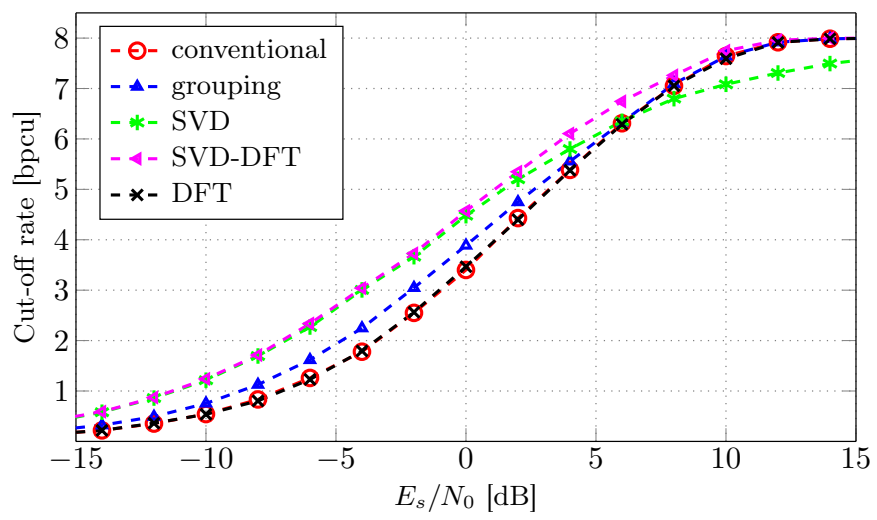

(a) $N_{a}=1$ and $M=32$.

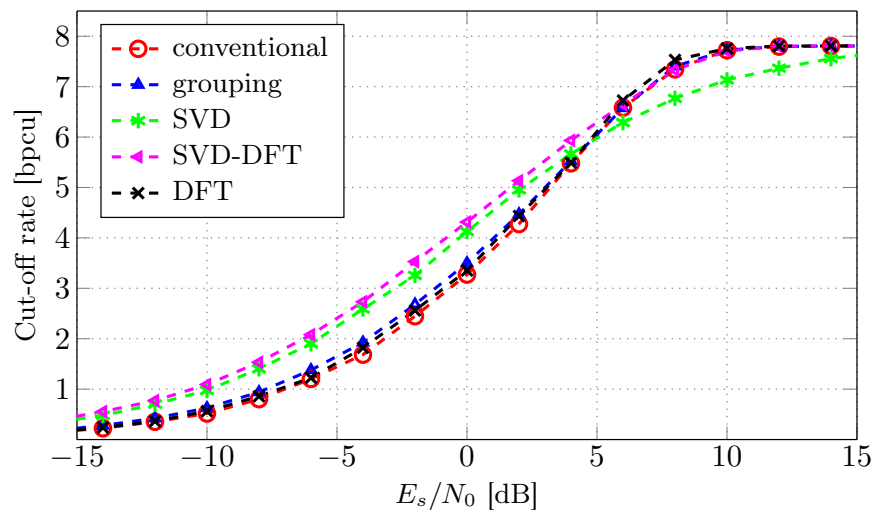

(b) $N_{a}=2$ and $M=8$.

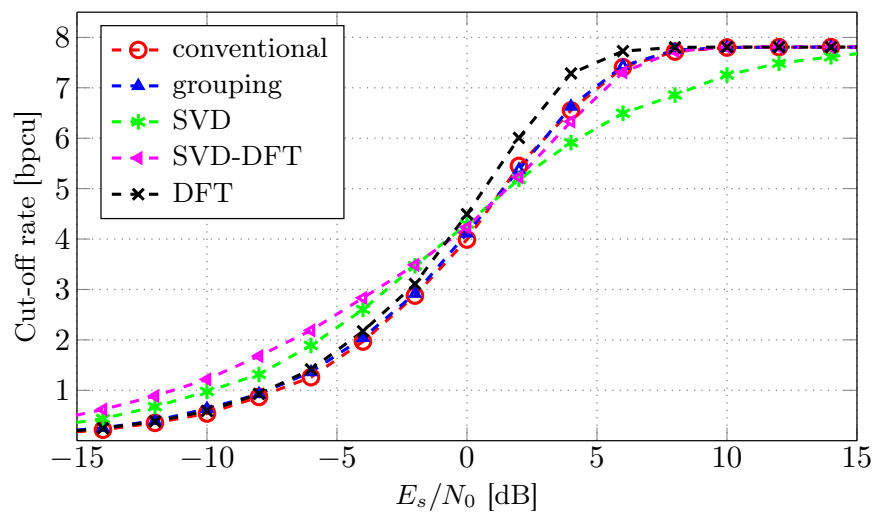

(c) $N_{a}=3$ and $M=4$.

Fig. 2. Cut-off rate of conventional GSM and GSM with the proposed precoding schemes in an i.i.d. Rayleigh fading channel.

This setup ensures, in fact, that the largest array gain is obtained but at the cost of having no signal transmission in the spatial domain. DFT precoding, on the other hand, is based on a fundamentally different operating principle. Its performance is predominately determined by the order of the IQ constellation, i.e., $M$. By using DFT precoding, the largest improvement of cut-off rate is obtained by decreasing $M$. If high-order IQ modulation alphabets are used, the minimum Euclidean distance between different RX-side GSM symbols is determined by the minimum Euclidean distance of the IQ constellation. So, a large gain in cut-off rate is usually not 


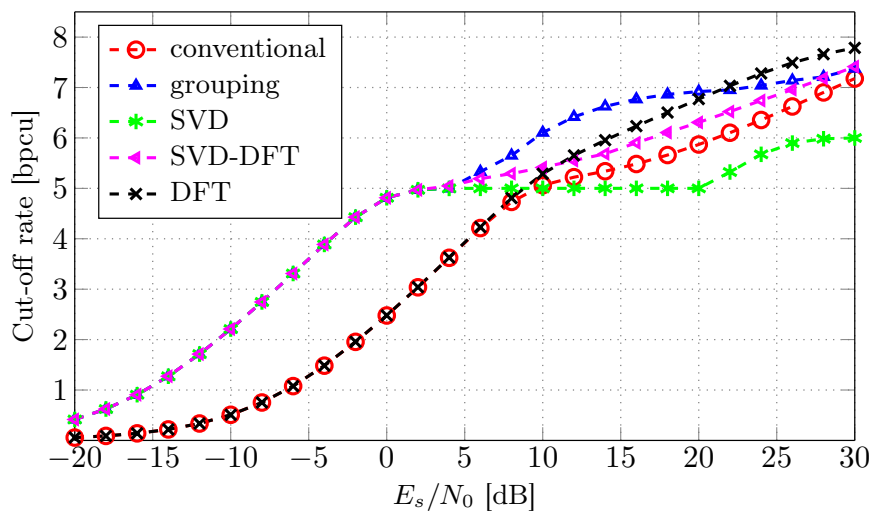

(a) $N_{a}=1$ and $M=32$.

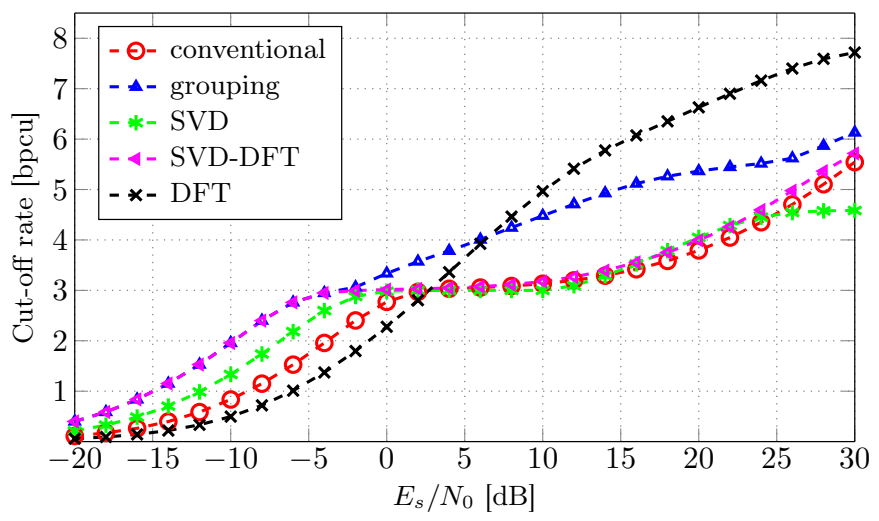

(b) $N_{a}=2$ and $M=8$.

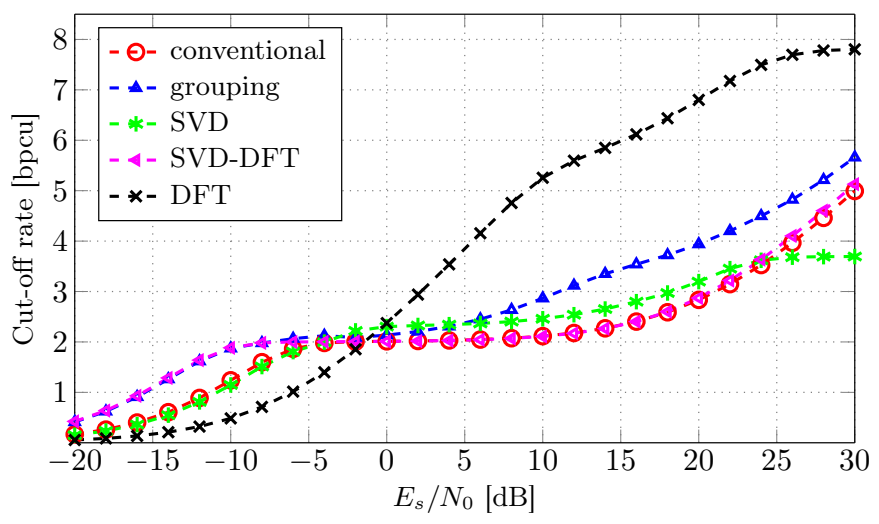

(c) $N_{a}=3$ and $M=4$.

Fig. 3. Cut-off rate of conventional GSM and GSM with the proposed precoding schemes in a pure LOS channel.

expected. If low-order modulation alphabets are utilized, on the other hand, the DFT precoding scheme is expected to provide a larger gain because it aims to maximize the minimum Euclidean distance between RX-side GSM symbols with different TX antenna activation patterns, disregarding the array gain. Thus, it can not improve cut-off rate at low SNR and it is more suitable for environments with correlated channels. So, it provides only a limited cut-off rate improvement in i.i.d. Rayleigh fading channels.

2) Cut-Off Rate in Pure LOS Channels: In Fig. 3, the cut-off rate of conventional GSM systems and the proposed precoding schemes over a pure LOS channel is illustrated. In low SNR regime, we observe that the cut-off rate of conventional GSM increases by increasing $N_{a}$. This is due to the fact that in correlated fading channels, e.g., pure LOS channels, the activation of several TX antennas that emit the same IQ symbol increases the array gain, which in turn increases cut-off rate. In medium to high SNR regime, on the other hand, channel models with high spatial correlation cause a reduction of the cut-off rate of conventional GSM systems as a function of $N_{a}$. The first three precoding schemes offer a linear increase of cut-off rate up to $\log _{2} M$ in low SNR regime. While SVD and SVD-DFT precoding schemes operate with $N_{a}$ active inputs and the TX antenna grouping precoding scheme has $N_{a}$ virtual antennas, no bits are encoded into the spatial domain in order to achieve higher array gain. In low SNR regime, SVD-DFT precoding provides always equal or better cut-off rate than SVD precoding, because of its ability to balance the power gains of the selected inputs, i.e., subchannels (see Lemma 2). It is worth noting that, in medium SNR regime, SVD-DFT precoding may give lower cut-off rate than SVD precoding, which can be explained by the following argumentation. At medium SNR, SVD-DFT precoding (same as SVD and TX antenna grouping precoding) starts to transmit data in spatial domain and therefore we have $N_{e}>N_{a}$. While this happens at almost the same SNR range for all $N_{a}$, it is safe to state that the number of the selected precoding matrix inputs $N_{e}$ for SVD-DFT precoding increases with $N_{a}$ at fixed SNR value. Due to high correlation of $\mathbf{H}$, we have $\lambda_{1} \gg \lambda_{2}, \ldots, \lambda_{N_{e}}$ and after a few derivation steps we can show that all columns of $\mathbf{G}_{e}$ are equal to $\mathbf{G}_{e}(:, i)=\left(\lambda_{1} \mathbf{U}(:, 1)+\mathbf{r}_{i}\right) / \sqrt{N_{e}}, i \in\left\{1, \ldots, N_{e}\right\}$, where $\mathbf{U}(:, 1)$ is a left-singular vector of $\mathbf{H}$ and $\mathbf{r}_{i} \in \mathbb{C}^{N_{r} \times 1}$ is a column vector, whose elements have absolute values close to 0 . Hence, if $N_{a}$, and consequently $N_{e}$, increases, the Euclidean distance between different columns of $\mathbf{G}_{e}$, which is equal to $\| \mathbf{G}_{e}(:, i)-\mathbf{G}_{e}\left(:, j\left\|^{2}=\right\| \mathbf{r}_{i}-\mathbf{r}_{j} \|^{2} / N_{e},(i \neq j)\right.$, generally decreases, making it harder to detect the activated inputs (i.e., columns) of $\mathbf{G}_{e}$ correctly. As a result, SVD-DFT precoding can offer a slightly lower cut-off rate than SVD precoding for $N_{a}=3$ in medium SNR regime. Another thing worth noting is that in high SNR regime $(>20 \mathrm{~dB})$, SVD precoding provides a cut-off rate that is even worse than conventional GSM systems. This effect also occurs in i.i.d. Rayleigh channel and it is caused by the limited number of the selected precoding matrix inputs, i.e., the number of GSM symbols. Moreover, in medium to high SNR regime, TX antenna grouping precoding offers a larger cut-off rate than SVD and SVD-DFT precoding. DFT precoding, on the other hand, provides the largest improvement of cut-off rate in high SNR regime. This is obtained thanks to its capability of increasing the minimum Euclidean distance between GSM symbols with different TX antenna activation patterns, which is particularly affected by the large channel correlation. The resulting improvement is inversely proportional to $M$ and it is greater than $3 \mathrm{bpcu}$ if $M=4$. Similar to the i.i.d. Rayleigh fading case, on the other hand, DFT precoding in unable to provide good cut-off rate in low SNR regime because it is not conceived for achieving a large array gain. More precisely, an 
increase of the minimum Euclidean distance between GSM symbols can reduce the array gain in a pure LOS channel and thus GSM with DFT precoding achieves even lower cut-off rate than conventional GSM in low SNR regime.

\section{B. Comparison With the Mutual Information of SM/GSM}

As discussed in Section I, cut-off rate constitutes a lowerbound of channel capacity. Thus, it is important to analyze to what extend improving cut-off rate results into a corresponding improvement of mutual information. The mutual information of GSM, based on the system model (1), can be calculated by using the analytical framework in [24], [25], as follows:

$$
\begin{aligned}
& \mathcal{I}\left(s_{m}, \mathbf{e}_{i} ; \mathbf{y}\right)=\log _{2} N_{\mathrm{GSM}}-\frac{1}{N_{\mathrm{GSM}}} \sum_{m, i} \times \\
& \mathbb{E}_{\mathbf{n}}\left\{\log _{2}\left[\sum_{n, j} \exp \left(-\frac{\left\|\mathbf{d}_{m, i}^{n, j}+\mathbf{n}\right\|^{2}-\|\mathbf{n}\|^{2}}{N_{0}}\right)\right]\right\}
\end{aligned}
$$

where $m, n=1, \ldots, M$ and $i, j=1, \ldots, N_{c}$. The difference vector of two GSM symbols at the RX is equal to $\mathbf{d}_{m, i}^{n, j}=\mathbf{H}\left(s_{m} \mathbf{e}_{i}-s_{n} \mathbf{e}_{j}\right) / \sqrt{N_{a}}$. It is worth mentioning that the mutual information in (35) corresponds to the channel capacity of GSM systems over a discrete-input continuous-output memoryless channel (DCMC) with uniformly distributed GSM symbols [35]. Hence, (35) can be used to calculate and compare the mutual information of conventional GSM systems and GSM systems with the proposed precoding schemes.

Mutual information is illustrated in Fig. 4. Similar to the previous case study, i.i.d. Rayleigh fading and pure LOS channel models are studied by keeping the same simulation setup. In low SNR regime, for both channel models, SVD and SVDDFT precoding schemes outperform, conventional GSM systems. TX antenna grouping offers a substantial improvement of mutual information in pure LOS channels and almost no gain in i.i.d. Rayleigh fading channels. DFT precoding improves mutual information in medium to high SNR regime, especially for LOS channels. We observe that mutual information and cut-off rate provide similar performance trends. Quantitatively, the gains are similar as well, but the gains observed for mutual information are slightly smaller than those observed for cut-off rate. This similarity can be justified with the aid of the lowerbound expression of the mutual information of GSM systems that is obtained by correcting the previous derivation in [40] (see Appendix B). The final result is as follows:

$$
\mathcal{I}_{\mathrm{LB}}=N_{r}\left(1-\log _{2} e\right)+R_{0}\left(N_{0} / 2\right),
$$

where $R_{0}\left(N_{0} / 2\right)$ corresponds to the cut-off rate in (4) by reducing the noise power by half (thus doubling SNR). Of course, the expression of the mutual information in (36) is well defined for values of the SNR for which it is positive. Equation (36), even though it may be considered a very crude approximation for mutual information, distinctly and explicitly highlights the dependency between cut-off rate and mutual information. In particular, it shows that an increase of cut-off rate results in an increase of mutual information as well. In summary, (36) and the numerical illustrations in Fig. 4 justify

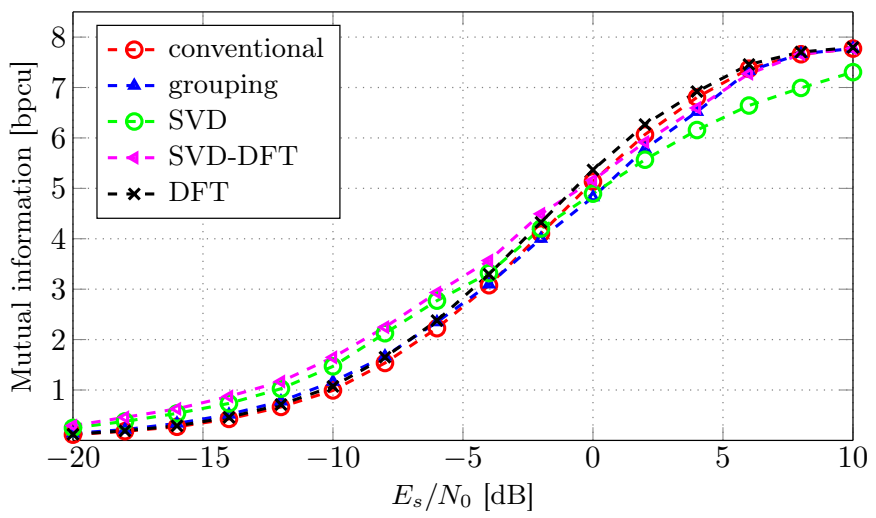

(a) I.i.d. Rayleigh channel.

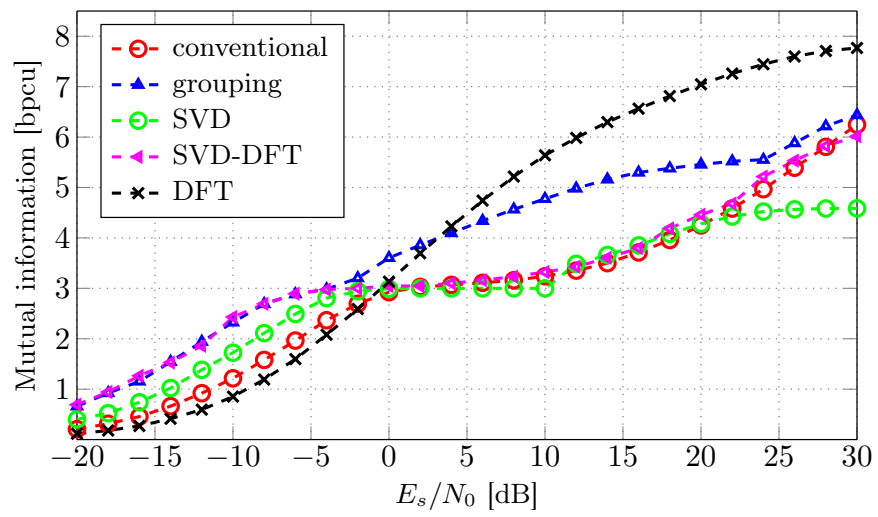

(b) Pure LOS channel.

Fig. 4. Mutual information of conventional GSM systems and GSM systems with the proposed precoding schemes in an i.i.d. Rayleigh channel and a pure LOS channel. The setup of parameters is: $N_{t}=N_{r}=8, N_{a}=2$ and $M=8$.

the choice of cut-off rate as a meaningful metric for optimizing the mutual information of GSM systems with precoding.

\section{Channel Capacity of GSM With Gaussian Signaling}

In this section, we analyze channel capacity gains achieved by using the proposed precoding schemes when discrete IQ signaling is substituted by continuous Gaussian signaling. More precisely, after determining the best precoding scheme configuration for GSM with discrete IQ signaling, channel capacity is computed by assuming that IQ symbols are distributed according to $\mathcal{C N}(0,1)$, i.e., Gaussian signaling. The channel capacity analytical expression is presented in [10] and it is the sum of IQ domain and spatial domain capacities.

The simulation results of the channel capacity of conventional GSM and GSM with the proposed precoding schemes are shown in Fig. 5. In a i.i.d. Rayleigh channel, the achieved gains in channel capacity are similar to the gains achieved for mutual information and cut-off rate. On the other hand, capacity gains in pure LOS channels are not very predictable. The channel capacity curves of the first three precoding schemes exhibit non-linear changes, when they start encoding information in spatial domain. In that situation, the number of the selected inputs for SVD and SVD-DFT precoding schemes is more than $N_{a}$, and TX antenna grouping precoding uses 
more than $N_{a}$ virtual TX antennas. So the maximum array gain is not achieved for every GSM symbol. As a result, IQ domain capacity may just slightly increase or even decrease, and spatial domain capacity is not large enough to maintain linearity of the channel capacity curves. SVD and SVD-DFT precoding schemes offer capacity gains in almost the whole SNR range, except in high SNR regime, where SVD precoding does not provide any gain. This is particularly visible for SVDDFT precoding schemes, which provides the best capacity performances among all the proposed precoding schemes. As expected, TX antenna grouping provides capacity gains over the whole SNR range. Because performance gains of DFT precoding are inversely proportional to the order of the IQ constellation [38] and Gaussian signaling can be viewed as an IQ constellation with infinite order, DFT precoding is unable to achieve any capacity gain in Fig. 5 .

\section{Comparison to Spatial Multiplexing (SMX)}

In this section, we focus our attention on comparing the cut-off rate of GSM systems with and without the proposed precoding schemes against its counterpart for SMX systems. To make the comparison fair and meaningful, the same precoding schemes used for GSM systems are applied to conventional SMX systems as well. More precisely, the RX signal vector of a $N_{t} \times N_{r}$ conventional SMX system can be written as follows:

$$
\mathbf{y}=\frac{1}{\sqrt{N_{t}}} \mathbf{H} \mathbf{s}_{m}+\mathbf{n}
$$

where the SMX symbol $\mathbf{s}_{m}=\left[s_{1} \ldots s_{N_{t}}\right]^{\mathrm{T}}$ is a column vector consisting of $N_{t}$ IQ symbols that are chosen from a symbol alphabet of $M$ elements. We assume a normalized symbol alphabet, i.e., $\mathbb{E}\left\{\left|s_{i}\right|^{2}\right\}=1$. By using the same reasoning as for GSM systems, the cut-off rate of a SMX system with equiprobable IQ symbols can be formulated as follows:

$$
R_{0}=-\log _{2}\left[\frac{1}{M^{2 N_{t}}} \sum_{m, n} \exp \left(-\frac{\left\|\mathbf{H}\left(\mathbf{s}_{m}-\mathbf{s}_{n}\right)\right\|^{2}}{4 N_{0} N_{t}}\right)\right]
$$

where $m, n=1, \ldots, M^{N_{t}}$. The cut-off rate of SMX systems employing the precoding schemes introduced in Section III can be obtained in a similar manner as for GSM systems. Due to space limitations, the details are omitted for brevity. The only exception is DFT precoding, which cannot be used for SMX systems. This is due to the fact that it is not possible to change the TX antennas that are activated for different SMX symbols.

We consider an $8 \times 8$ SMX-MIMO system. In order to obtain the same bpcu as for GSM systems, i.e., $8 \mathrm{bpcu}$, $M=2$ is considered. The numerical results in Fig. 6 show that conventional SMX outperforms conventional SM in i.i.d. Rayleigh fading channels, because different IQ steams can be easily resolved at the RX. This confirms early results on the performance of SMX systems about the suitability of this modulation scheme for uncorrelated fading channels. In highly correlated channels, i.e., pure LOS channels, the situation is opposite and conventional SM shows an advantage. In addition, we observe that the proposed precoding schemes

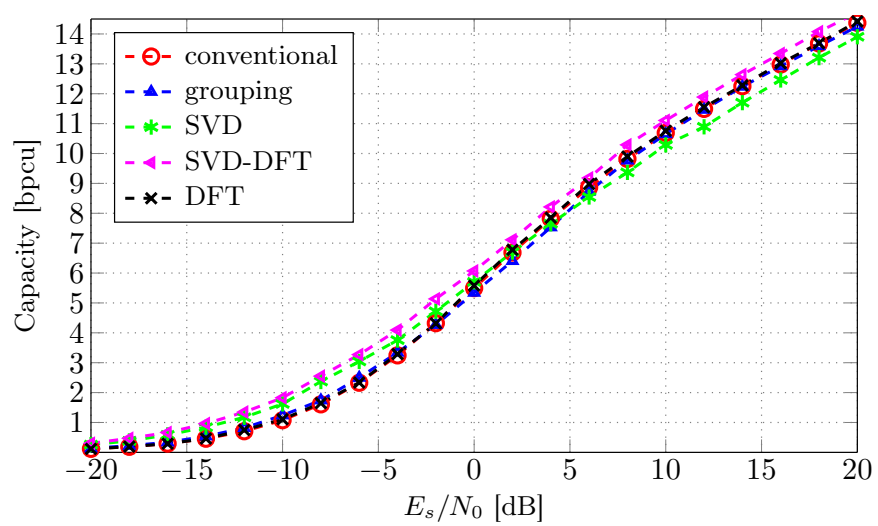

(a) I.i.d. Rayleigh channel.

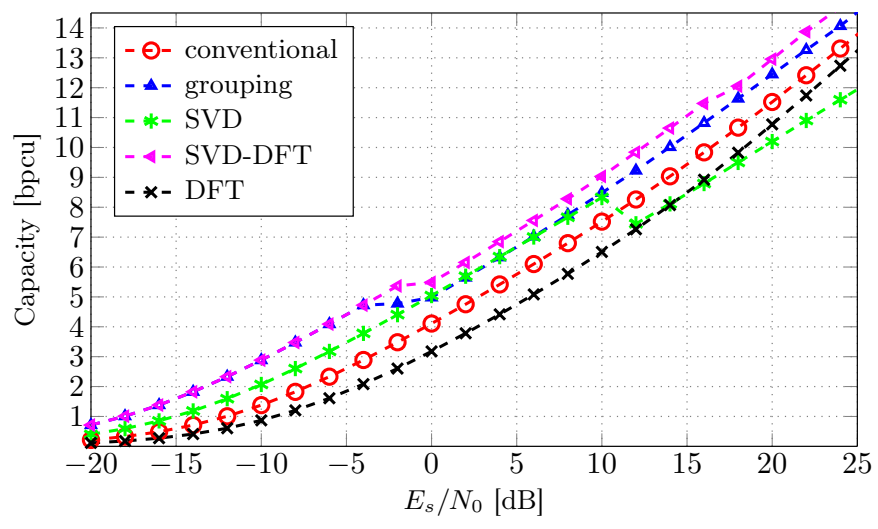

(b) Pure LOS channel.

Fig. 5. Channel capacity of conventional GSM and GSM with the proposed precoding schemes, assuming Gaussian signaling. The setup of parameters is the same as in Fig. 4.

enable cut-off rate increases which are, in general, larger for SM, especially in pure LOS channels. A reason for this performance trend lies in a larger reduction of the number of TX symbols for SMX systems as opposed to SM systems. For example, the largest reduction of the number of TX symbols occurs when SVD and SVD-DFT precoding schemes have only one active column in the precoding matrix, and just one virtual antenna is used for the TX antenna grouping precoding scheme. Then, the number of SM symbols is 16 times larger than the number of SMX symbols.

\section{E. Cut-off rate Optimization vs. SEP Optimization}

By direct inspection of (2), we note that the analytical expression of cut-off rate resembles the upper-bound of the SEP of GSM systems, which can be formulated as follows:

$$
\mathrm{SEP} \leq \frac{1}{N_{\mathrm{GSM}}} \sum_{\substack{m, n, i, j \\(m, i) \neq(n, J)}} \exp \left(-\frac{\left\|\mathbf{H}\left(\mathbf{e}_{i} s_{m}-\mathbf{e}_{j} s_{n}\right)\right\|^{2}}{4 N_{0} N_{a}}\right)
$$

where $m, n=1, \ldots, M$ and $i, j=1, \ldots, N_{c}$. This similarity motivates us to study the effectiveness of maximizing cut-off rate for increasing the mutual information of GSM systems as opposed to minimizing the SEP. To this end, we optimize 


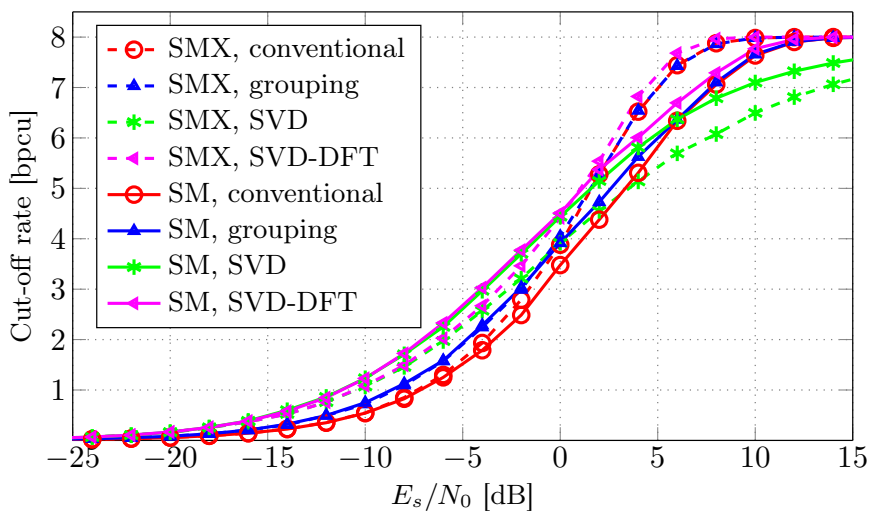

(a) I.i.d. Rayleigh channel.

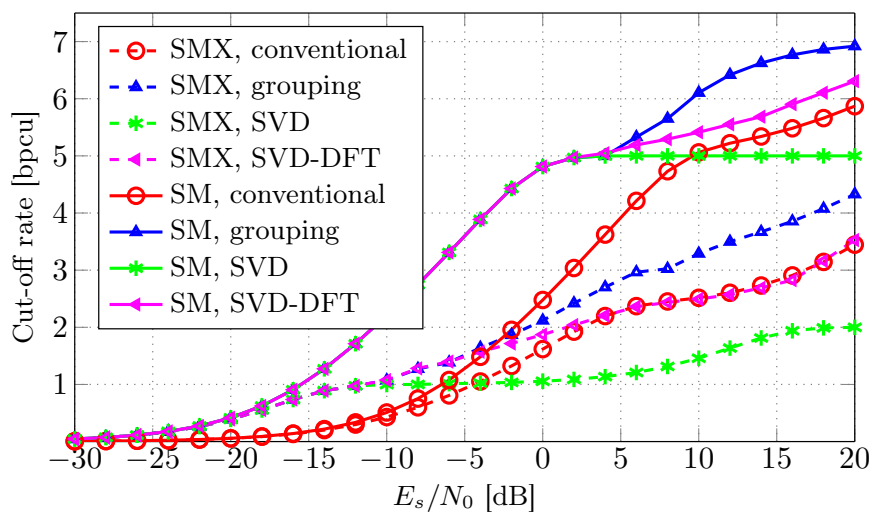

(b) Pure LOS channel.

Fig. 6. Cut-off rate comparison between SMX and SM systems (both systems employ the proposed precoding schemes).

the proposed precoding schemes based on the minimization of SEP rather than on the maximization of cut-off rate. In other words, Algorithms 1, and 2 still apply and the only modification consists of replacing the argument of the logarithm function of cut-off rate with (39). The only scheme that is not considered is DFT precoding, since in this case maximizing cut-off rate and minimizing SEP are equivalent. This is not the case, on the other hand, for the other precoding schemes.

The results are illustrated in Fig. 7. We observe that, in the medium to high SNR regime, optimizing cut-off rate results in a larger mutual information compared to optimization by SEP. The reason for this performance trend lies in the larger number of GSM symbols that results from optimizing cut-off rate as opposed to optimizing SEP (Appendix C). In a nutshell, the following approximation holds true in high SNR regime $\left\|\mathbf{d}_{m, i}^{n, j}+\mathbf{n}\right\|^{2}-\|\mathbf{n}\|^{2} \approx\left\|\mathbf{d}_{m, i}^{n, j}\right\|^{2}$ and the mutual information in (35) can be re-written as follows:

$$
\begin{aligned}
& \mathcal{I}\left(s_{m}, \mathbf{e}_{i} ; \mathbf{y}\right) \approx \log _{2} N_{\mathrm{GSM}}-\frac{1}{N_{\mathrm{GSM}}} \sum_{m, i} \\
& \log _{2}\left[\sum_{n, j} \exp \left(-\left\|\mathbf{d}_{m, i}^{n, j}\right\|^{2} / N_{0}\right)\right] .
\end{aligned}
$$

In (40), in high SNR regime, the exponential terms that satisfy the condition $(m, i) \neq(n, j)$ approach 0 . This implies that the

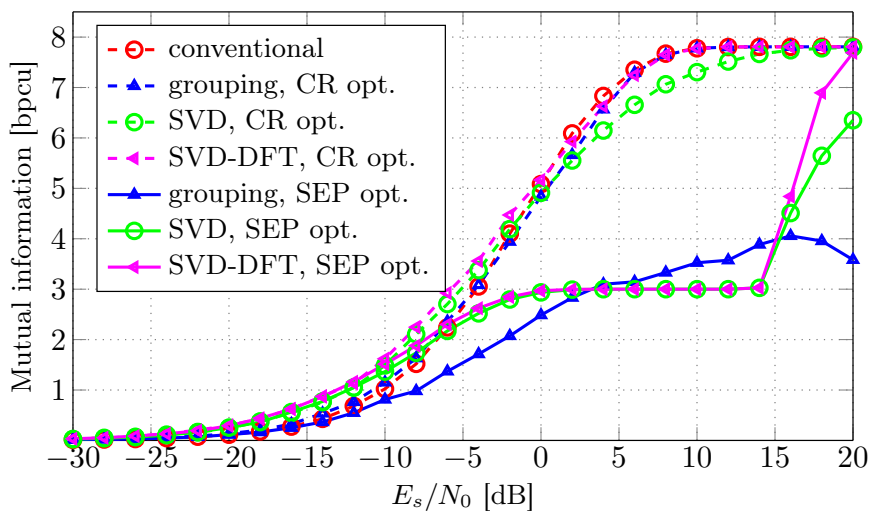

(a) I.i.d. Rayleigh channel.

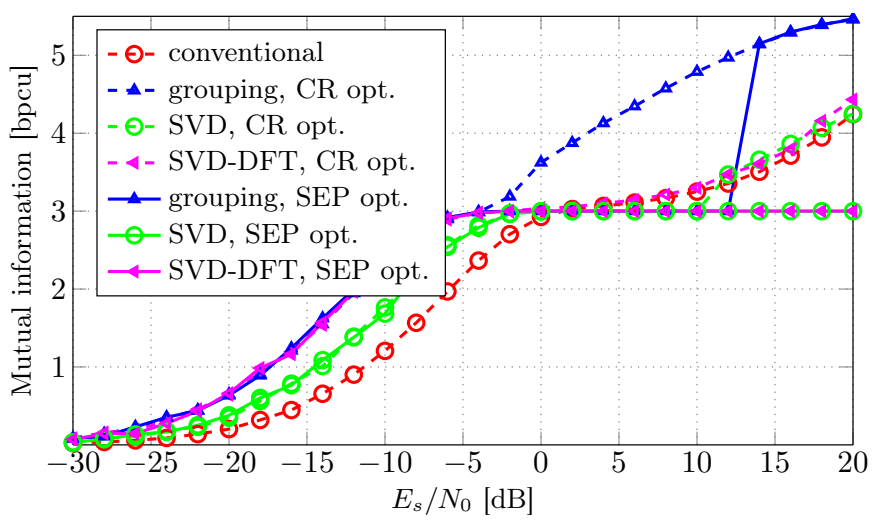

(b) Pure LOS channel.

Fig. 7. Mutual information of GSM with the proposed precoding schemes based on cut-off rate (CR) and SEP optimization. The setup of parameters is the same as in Fig. 4.

minimum Euclidean distance between different GSM symbols is a less important factor for maximizing mutual information as opposed to the number of GSM symbols available. Since optimizing the systems based on cut-off rate usually provides more GSM symbols, a better mutual information is expected as well. This performance trend becomes evident in i.i.d. Rayleigh fading channels at medium to high SNR, where the optimization of SEP results in a very small number of TX antenna activation patterns. Fig. 7 shows that optimizing SEP may lead to a mutual information that is worse than the benchmark that does not use precoding. In a pure LOS channel, the proposed precoding schemes based on either cutof rate or SEP as a utility function for optimization use only a subset of all the available TX antenna activation patterns (except in the very high SNR regime). This is due to the larger channel correlation between the columns of the channel matrix. As a result, the differences in mutual information are smaller. In low SNR regime, there is almost no difference between optimizing SEP and cut-off rate because they both result in maximizing the array gain. Hence, a small (usually minimum) number of TX antenna activation patterns is used.

Furthermore, it is worth noting that SEP optimization as shown in Fig. 7 may experience a sudden increase of mutual information in high SNR regime, due to a rapid increase of 
the number of GSM symbols. The increase of the number of GSM symbols also enlarges the sum of the exponential terms in (4) and (39), which has a negative influence on SEP and cut-off rate, and is inversely proportional to SNR. As the argument of the logarithm function of cut-rate rate is proportional to $1 / N_{\mathrm{GSM}}^{2}$ and SEP is proportional to $1 / N_{\mathrm{GSM}}$, SEP optimization requires a larger SNR than cut-off rate optimization, so that the increase of the number of GSM symbols has a "more decisive" influence than the proper increase of the sum of the exponential terms. Hence, the number of GSM symbol obtained by SEP optimization starts to increase rapidly only at high SNR, which results in sharply rising mutual information curves.

\section{F. Application to QSM Systems}

In this section, we extend our study to apply the proposed precoding schemes, which are originally designed for GSM, to QSM. The RX signal for QSM is given as follows [41]:

$$
\mathbf{y}=\mathbf{h}_{i_{1}} s_{m, R}+\mathbf{j h}_{i_{2}} s_{m, I}+\mathbf{n},
$$

where $s_{m}=s_{m, R}+\mathbf{j} s_{m, I}(m \in\{1, \ldots, M\})$ is the transmitted IQ symbol. The TX antennas that transmit $s_{m, R}$ and $s_{m, I}$ have impulse responses $\mathbf{h}_{i_{1}}$ and $\mathbf{h}_{i_{2}}\left(i_{1}, i_{2} \in\left\{1, \ldots, N_{t}\right\}\right)$, respectively, and they determine the $i$-th QSM TX antenna activation pattern. Following a similar derivation as in Section II-A, we obtain the cut-off rate expression for QSM as follows:

$$
R_{0}=-\log _{2}\left[\frac{1}{N_{\mathrm{QSM}}^{2}} \sum_{i_{1}, i_{2}, j_{1} j_{2}, m, n} \exp \left(-\frac{\Phi}{4 N_{0}}\right)\right],
$$

where $i_{1}, i_{2}, j_{1}, j_{2} \in\left\{1, \ldots, N_{t}\right\}$ and $m, n \in\{1, \ldots, M\}$. The Euclidean distance between two QSM symbols is computed as

$$
\Phi=\left\|\left(\mathbf{h}_{i_{1}} s_{m, R}+\mathbf{j h}_{i_{2}} s_{m, I}\right)-\left(\mathbf{h}_{j_{1}} s_{n, R}+\mathbf{j} \mathbf{h}_{j_{2}} s_{n, I}\right)\right\|^{2}
$$

and the number of QSM symbols is $N_{\mathrm{QSM}}=M N_{t}^{2}$. In our analysis we assume that the number of TX antennas for the transmission of $s_{m, R}$ is always equal to the number of TX antennas for the transmission of $s_{m, I}{ }^{5}$. The same is valid for virtual TX antennas (TX antenna grouping precoding) and selected precoding matrix inputs (SVD and SVD-DFT precoding). The first three precoding schemes can be directly applied to QSM, and the cut-off rate expression for QSM needs just a minor adaptation in the argument of the logarithm function. On the other hand, DFT precoding requires a larger modification, in order to be applicable to QSM systems. The RX signal for QSM with DFT precoding, when the $i$-th QSM TX antenna activation pattern is used, is given by $\mathbf{y}=\mathbf{h}_{i_{1}} \tilde{\mathbf{P}}(1, i) s_{m, R}+$ $\mathbf{j h}_{i_{2}} \tilde{\mathbf{P}}(2, i) s_{m, I}+\mathbf{n}$, where the only addition in comparison to (41) is the precoding matrix $\tilde{\mathbf{P}} \in \mathbb{C}^{2 \times N_{t}^{2}}$. Each column of $\tilde{\mathbf{P}}$ is a precoding vector that contains two precoding coefficients

\footnotetext{
${ }^{5}$ We do not restrict the number of TX antennas for transmission of $s_{m}, R$ and $s_{m, I}$ to be power of 2 . While QSM can be viewed as a combination of two independent SM systems (one that transmits $s_{m, R}$ and the other that transmits $\left.s_{m, I}\right)$, an implementation of so-called fractional bit modulation method [31] on both of them may enable the use of all available TX antennas (i.e., noninteger data rate) in QSM systems.
}

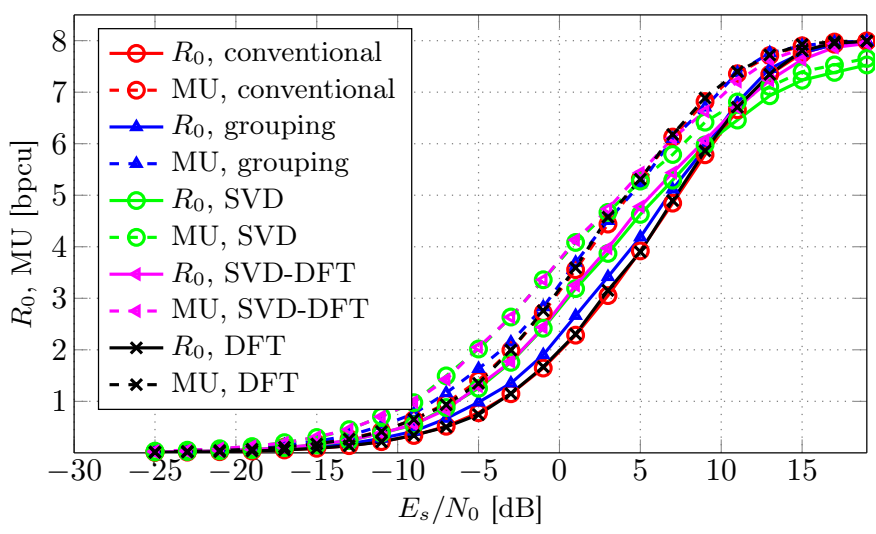

(a) I.i.d. Rayleigh channel.

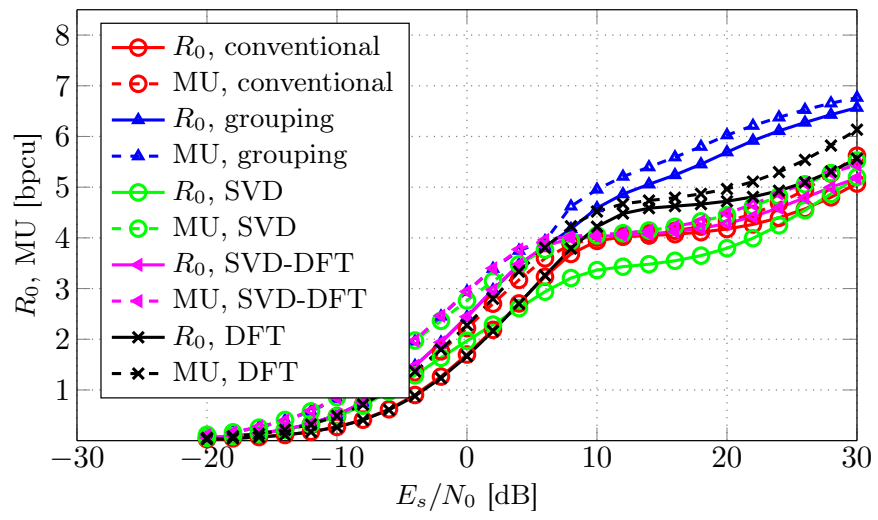

(b) Pure LOS channel.

Fig. 8. Cut-off rate $\left(R_{0}\right)$ and mutual information (MU) of conventional QSM systems and QSM systems with the proposed precoding schemes.

for the active TX antennas that transmit $s_{m, R}$ and $s_{m, I}$, and are determined by an appropriate QSM TX antenna activation pattern. If one TX antenna transmits simultaneously $s_{m, R}$ and $s_{m, I}$ for one QSM TX antenna activation pattern then both precoding coefficients of an appropriate precoding vector have the same value. Similarly as in Algorithm 3, matrix $\tilde{\mathbf{P}}$ is empty at the beginning and $N_{t}^{2}$ iterations are needed to form its content. In each iteration, the candidate precoding vectors $\mathbf{t}_{k} \in \mathbb{C}^{2 \times 1}\left(k \in\left\{1, \ldots, N_{t}\right\}\right)$ for the $i$-th QSM TX antenna activation pattern are formed from the rows of the DFT matrix $\mathbf{W}$ that have the same indices as the active TX antennas. After constructing all $\tilde{\mathbf{P}}_{k}$ matrices, as in Algorithm 3, we select the one with the largest minimum Euclidean distance. Due to the constant increase of the size of $\tilde{\mathbf{P}}$, the Euclidean distances are computed as $\tilde{\Phi}^{\prime}=\| \mathbf{h}_{i_{1}} \tilde{\mathbf{P}}_{k}(1, i) s_{m, R}+\mathbf{j} \mathbf{h}_{i_{2}} \tilde{\mathbf{P}}_{k}(2, i) s_{m, I}-$ $\left(\mathbf{h}_{j_{1}} \tilde{\mathbf{P}}_{k}(1, j) s_{n, R}+\mathbf{j} \mathbf{h}_{j_{2}} \tilde{\mathbf{P}}_{k}(2, j) s_{n, I}\right) \|^{2}$, where $i_{1}$ and $i_{2}$ are the indices of the active TX antennas for the $i$-th QSM TX antenna activation pattern, $j_{1}$ and $j_{2}$ are the indices of the active TX antennas for the $j$-th $(j=1, \ldots, i)$ QSM TX antenna activation pattern, and $m, n \in\{1, \ldots, M\}$. After $N_{t}^{2}$ iterations, the content of $\tilde{\mathbf{P}}$ is determined and the cut-off rate of QSM with DFT precoding can be calculated.

Performances of the proposed precoding schemes applied to QSM systems are evaluated using cut-off rate and mutual information. The mutual information expression for QSM 
presents just a slight modification of (35), where $N_{\mathrm{GSM}}$ is substituted by $N_{\mathrm{QSM}}$ and the difference vector of two RX-side QSM symbols is $\mathbf{d}=\left(\mathbf{h}_{i_{1}} s_{m, R}+\mathbf{j} \mathbf{h}_{i_{2}} s_{m, I}\right)-\left(\mathbf{h}_{j_{1}} s_{n, R}+\right.$ $\left.\mathbf{j h}_{j_{2}} s_{n, I}\right)$. Also, repeating a derivation from [35], we can easily show that the mutual information expression for QSM corresponds to the capacity expression for QSM in DCMC channels, when QSM symbols are uniformly distributed. While QSM generally requires only one RF chain at the TX same as SM, we evaluate the mutual information of QSM under the same date rate of $8 \mathrm{bpcu}$, and for a balanced data rate in the spatial and IQ domains as for SM (although the spatial and IQ domain data rates are not exactly the same for SM). Hence, the simulation setup consists of a $4 \times 4$ MIMO system with 16-QAM and the simulation results are presented in Fig. 8. The simulation results show that the proposed precoding schemes applied to QSM systems achieve mutual information gains that are very similar to cut-off rate gains. This confirms the applicability of cut-off rate as an optimization metric for QSM as well.

\section{CONCLUSION}

In this paper, we proposed the use of cut-off rate as a simple and meaningful utility function for optimizing the channel capacity and mutual information of SM/GSM systems. Also, we proposed four precoding schemes whose precoding matrices are obtained by maximizing cut-off rate. Numerical results confirmed that they provide a better mutual information and channel capacity than conventional SM/GSM systems. More precisely, we showed that the precoding schemes that aim to maximize array gain are more suitable for application in low to medium SNR regime. On the other hand, the precoding schemes that aim to maximize the minimum Euclidean distance between SM/GSM symbols, when discrete IQ signaling is utilized, are more suitable for application in medium to high SNR regime. In summary, our study confirmed that cut-off rate is a suitable metric for the design of precoding schemes that optimize the channel capacity and mutual information of SM/GSM. Depending on the operating SNR, different precoding schemes need to be used in order to achieve the best channel capacity and mutual information. Possible extension of the present work may be a joint optimization of coding and precoding, which due to space constrains, is postponed to future research.

\section{APPENDIX A}

\section{Cut-off Rate of TX Antenna Grouping Precoding}

In this appendix, we analyze the improvement cut-off rate that TX antenna grouping precoding is capable of providing in i.i.d. Rayleigh fading channels and pure LOS channels. For ease of description, only groups of two antennas as considered. Similar findings and trends hold for a large number of antenna in each group. Let $\mathbf{h}_{i}$ and $\mathbf{h}_{j}\left(i, j \in\left\{1, \ldots, N_{t}\right\}, i \neq j\right)$ be the impulse responses of two physical TX antennas. Let us denote by $\mathbf{h}_{g}$ the virtual TX antenna after grouping them. The following holds:

$$
\mathbb{E}\left\{\left\|\mathbf{h}_{g}\right\|^{2}\right\}=\frac{1}{2} \mathbb{E}\left\{\left\|\mathbf{h}_{i}\right\|^{2}+\left\|\mathbf{h}_{j}\right\|^{2}+2 \Re\left\{\mathbf{h}_{i}^{\mathrm{H}} \mathbf{h}_{j}\right\}\right\} .
$$

In i.i.d. Rayleigh fading channels, the mean value of the inner product of two different columns of $\mathbf{H}$ is equal to 0 , i.e., $\mathbb{E}\left\{\mathfrak{R}\left\{\mathbf{h}_{i}^{\mathrm{H}} \mathbf{h}_{j}\right\}\right\}=0$. In addition, we have $\mathbb{E}\left\{\left\|\mathbf{h}_{i}\right\|^{2}\right\}=N_{r}$ for $\forall i \in\left\{1, \ldots, N_{t}\right\}$. Thus, (43) simplifies to

$$
\mathbb{E}\left\{\left\|\mathbf{h}_{g}\right\|^{2}\right\}=N_{r},
$$

and the average power gain is the same as without grouping the physical antennas. This implies that the TX antenna grouping precoding scheme provides, in general, a relatively small improvement of cut-off rate in i.i.d. Rayleigh fading channels.

In pure LOS channels, on the other hand, we have $d_{t}, d_{r} \ll D$, i.e., the columns of $\mathbf{H}$ are highly correlated. This implies $\mathbb{E}\left\{\mathfrak{R}\left\{\mathbf{h}_{i}^{\mathrm{H}} \mathbf{h}_{j}\right\}\right\}>0$. In this regime, the approximation $\left\|\mathbf{h}_{i}\right\|^{2} \approx N_{r}$ for $\forall i \in\left\{1, \ldots, N_{t}\right\}$ holds true (cf. (34)) and (43) simplifies to

$$
\mathbb{E}\left\{\left\|\mathbf{h}_{g}\right\|^{2}\right\}>N_{r}
$$

In pure LOS channels, therefore, the TX antenna grouping precoding scheme increases the average power gain and, as a result, it is expected to provide a large improvement of cut-off rate as opposed to the i.i.d. Rayleigh fading case.

\section{APPENDIX B \\ LOWER-BOUND OF MUTUAL INFORMATION}

In this appendix, we present the corrected derivation of a lower-bound of the GSM mutual information from [40]. The mutual information of a GSM system is defined as follows:

$$
\mathcal{I}\left(s_{m}, \mathbf{e}_{i} ; \mathbf{y}\right)=\mathcal{H}(\mathbf{y})-\mathcal{H}\left(\mathbf{y} \mid s_{m}, \mathbf{e}_{i}\right)=\mathcal{H}(\mathbf{y})-\mathcal{H}(\mathbf{n})
$$

By using Jensen's inequality, we obtain:

$$
\mathcal{H}(\mathbf{y})=\mathbb{E}_{\mathbf{y}}\left\{-\log _{2}(p(\mathbf{y}))\right\} \geq-\log _{2}\left(\int_{\mathbf{y}} p(\mathbf{y})^{2} \mathrm{~d} \mathbf{y}\right)
$$

In addition, we have:

$p(\mathbf{y})=\sum_{m, i} p\left(s_{m}, \mathbf{e}_{i}\right) p\left(\mathbf{y} \mid s_{m}, \mathbf{e}_{i}\right)=\frac{1}{N_{\mathrm{GSM}}} \sum_{m, i} p\left(\mathbf{y} \mid s_{m}, \mathbf{e}_{i}\right)$,

where $p\left(\mathbf{y} \mid s_{m}, \mathbf{e}_{i}\right)$ is available in (3) and the GSM symbols are uniformly distributed.

By plugging (48) in (47) and applying some algebraic manipulations similar to the computation of the cut-off rate, we obtain the following:

$$
\begin{aligned}
\mathcal{H}(\mathbf{y}) \geq & N_{r} \log _{2}\left(2 \pi N_{0}\right)- \\
& \log _{2}\left(\frac{1}{N_{\mathrm{GSM}}^{2}} \sum_{m, n, i, j} e^{-\frac{\left\|\mathbf{H}\left(\mathbf{e}_{i} s_{m}-\mathbf{e}_{j} s_{n}\right)\right\|^{2}}{2 N_{0} N_{a}}}\right) \\
= & N_{r} \log _{2}\left(2 \pi N_{0}\right)+R_{0}\left(N_{0} / 2\right)
\end{aligned}
$$

for $m, n=1, \cdots, M$ and $i, j=1, \cdots, N_{c}$. From [14], we have $\mathcal{H}(\mathbf{n})=N_{r} \log _{2}\left(e \pi N_{0}\right)$ and eventually obtain the lowerbound reported in (36). 


\section{APPENDIX C \\ Number of GSM SyMBOLS OF THE PROPOSED \\ Precoding SCHEMES BASEd ON CUT-OFF RATE AND SEP OPTIMIZATION}

In this appendix, we discuss the reason why the precoding schemes that maximize cut-off rate instead of SEP result in a larger number of TX antenna activation patterns. First of all, we note that maximizing cut-off rate is equivalent to minimizing the argument of the logarithm function of cut-off rate, which is denoted as $\mathrm{AG}$ and related to SEP as follows:

$$
\mathrm{AG}=\frac{1}{N_{\mathrm{GSM}}}(1+\mathrm{SEP}) .
$$

Let us consider TX antenna grouping precoding. Let us assume that the number of TX antenna activation patterns decreases from $N_{\mathrm{GSM}}$ to $N_{\mathrm{GSM}}-\triangle N$ at an iteration of the algorithm and that the reduction of SEP is $\triangle \mathrm{SEP}>0$. Under these assumptions, the argument of the logarithm function of cutoff rate after optimizing SEP is given as follows:

$$
\mathrm{AG}_{1}=\frac{1}{N_{\mathrm{GSM}}-\triangle N}(1+\mathrm{SEP}-\triangle \mathrm{SEP})
$$

while before optimizing SEP, we have $\mathrm{AG}_{0}$ in (50).

If, on the other hand, optimizing the SEP does not reduce the argument of the logarithm function of the cut-off rate, we obtain:

$$
\triangle \mathrm{SEP}<\frac{\triangle N}{N_{\mathrm{GSM}}}(1+\mathrm{SEP}) .
$$

By taking into account that the number of virtual TX antennas at an iteration reduces by 1 , i.e., from $N_{v t}$ to $N_{v t}-1$, we have:

$$
\triangle \mathrm{SEP}<\frac{N_{a}}{N_{v t}}(1+\mathrm{SEP}) .
$$

If inequality (53) holds true, the number of TX antenna activation patterns obtained by optimizing cut-off rate is larger than the number of TX antenna activation patterns that is obtained by optimizing SEP. Otherwise, they result in the same number of TX antenna activation patterns. What is left to prove is to show that optimizing SEP cannot provide a larger number of TX antenna activation patterns compared to its counterpart that is obtained by maximizing cut-off rate. Assuming that at an iteration of the TX antenna grouping algorithm SEP increases by $\triangle \mathrm{SEP}>0$. Then, we have

$$
\mathrm{AG}_{1}=\frac{1}{N_{\mathrm{GSM}}-\triangle N}(1+\mathrm{SEP}+\triangle \mathrm{SEP})>\mathrm{AG}_{0}
$$

and the argument of the logarithm function of cut-off rate increases. Hence, the number of GSM symbols provided by SEP optimization cannot be larger than the the number of GSM symbols provided by cut-off rate optimization.

As far as SVD and SVD-DFT precoding schemes are concerned, the result can be proved based on the following argument. At each iteration of both algorithms, SEP is reduced by adding an additional column to the precoding matrix. This operation inherently decreases the argument of the logarithm function in (50) and, thus, increases cut-off rate. The opposite, on the other hand, is not always true. Therefore, optimizing the cut-off rate of GSM systems by using SVD and SVD-DFT precoding provides a larger (or the same in the worst case) number of TX antenna activation patterns than minimizing SEP.

\section{REFERENCES}

[1] R. Mesleh et al., "Spatial modulation - A new low complexity spectral efficiency enhancing technique," in 1st Int. Conf. on Communications and Networking in China, vol. 1, Oct. 2006, pp. 1-5.

[2] M. Di Renzo et al., "Spatial modulation for generalized MIMO: Challenges, opportunities, and implementation," Proc. IEEE, vol. 102, no. 1, pp. 56-103, Jan. 2014.

[3] E. Faregh et al., "A closed-form mutual information approximation for multiple-antenna systems with spatial modulation," IEEE Commun. Lett., vol. 5, no. 6, pp. 636-639, Dec. 2016.

[4] J. L. Massey, "Coding and modulation in digital communications," in Proc. of Int. Zurich Seminar, 1974, pp. E2.1-E2.4.

[5] G. Caire et al., "Bit-interleaved coded modulation," IEEE Trans. Inf. Theory, vol. 44, no. 3, pp. 927-946, May 1998.

[6] F. Rey et al., "Linear precoder design through cut-off rate maximization in MIMO-OFDM coded systems with imperfect CSIT,' IEEE Trans. Signal Process., vol. 58, no. 3, pp. 1741-1755, Mar. 2010.

[7] M. T. Ivrlac et al., "Fading correlations in wireless MIMO communication systems," IEEE J. Sel. Areas Commun., vol. 21, no. 5, pp. 819-828, Jun. 2003.

[8] J. Proakis and M. Salehi, Digital Communications, 5th ed. Boston: McGraw-Hill Science/Engineering/Math, 2007.

[9] R. U. Nabar et al., "Cut-off rate based transmit optimization for spatial multiplexing on general MIMO channels," in 2003 IEEE Int. Conf. on Acoustics, Speech, and Signal Processing, (ICASSP '03), vol. 5, Apr. 2003, pp. 61-64.

[10] P. Liu et al., "Line-of-sight spatial modulation for indoor mmWave communication at $60 \mathrm{GHz}$," IEEE Trans. Wireless Commun., vol. 15, no. 11, pp. 7373-7389, Nov. 2016.

[11] J. Wang et al., "Generalised spatial modulation system with multiple active transmit antennas and low complexity detection scheme," IEEE Trans. Wireless Commun., vol. 11, no. 4, pp. 1605-1615, Apr. 2012.

[12] A. Younis et al., "Generalised spatial modulation," in 2010 Conf. Rec. of the 44th Asilomar Conf. on Signals, Systems and Computers (ASILOMAR). IEEE, 2010, pp. 1498-1502.

[13] G. J. Foschini, "Layered space-time architecture for wireless communication in a fading environment when using multi-element antennas," Bell Labs Tech. J., vol. 1, no. 2, pp. 41-59, 1996.

[14] E. Telatar, "Capacity of multi-antenna gaussian channels," Eur. Trans. Telecommun., vol. 10, no. 6, pp. 585-595, Nov. 1999.

[15] A. Stavridis et al., "Energy evaluation of spatial modulation at a multiantenna base station," in Vehicular Tech. Conf. (VTC Fall), Sep. 2013 pp. $1-5$.

[16] R. Rajashekar et al., "Reduced-complexity ML detection and capacityoptimized training for spatial modulation systems," IEEE Trans. Commun., vol. 62, no. 1, pp. 112-125, Jan. 2014.

[17] Y. Yang and B. Jiao, "Information-guided channel-hopping for high data rate wireless communication," IEEE Commun. Lett., vol. 12, no. 4, pp. 225-227, Apr. 2008.

[18] P. Liu et al., "Variable- $N_{u}$ generalized spatial modulation for indoor LOS mmwave communication: Performance optimization and novel switching structure," IEEE Trans. Commun., vol. 65, no. 6, pp. 26252640, Jun. 2017.

[19] A. Younis et al., "Performance analysis for generalised spatial modulation," in Proc. 20th European Wireless Conf., May 2014, pp. 1-6.

[20] Z. An et al., "Mutual information analysis on spatial modulation multiple antenna system," IEEE Trans. Commun., vol. 63, no. 3, pp. 826-843, Mar. 2015.

[21] - "Mutual information and error probability analysis on generalized spatial modulation system," IEEE Trans. Commun., vol. 65, no. 3, pp. 1044-1060, Mar. 2017.

[22] P. Henarejos and A. I. Pérez-Neira, "Capacity analysis of index modulations over spatial, polarization, and frequency dimensions," IEEE Trans. Commun., vol. 65, no. 12, pp. 5280-5292, Dec. 2017.

[23] L. He et al., "On generalized spatial modulation aided millimeter wave MIMO: Spectral efficiency analysis and hybrid precoder design," IEEE Trans. Wireless Commun., vol. 16, no. 11, pp. 7658-7671, Nov. 2017.

[24] X. Guan et al., "On the mutual information and precoding for spatial modulation with finite alphabet," IEEE Commun. Lett., vol. 2, no. 4, pp 383-386, Aug. 2013. 
[25] S. R. Jin et al., "Linear precoding design for mutual information maximization in generalized spatial modulation with finite alphabet inputs," IEEE Commun. Lett., vol. 19, no. 8, pp. 1323-1326, Aug. 2015.

[26] X. Wang et al., "Spectral efficiency analysis for downlink noma aided spatial modulation with finite alphabet inputs," IEEE Trans. Veh. Technol., vol. 66, no. 11, pp. 10562-10566, Dec. 2017.

[27] A. Yadav et al., "Linear precoder design for correlated partially coherent channels with discrete inputs," in ISWCS 2013; The Tenth Int. Symp. on Wireless Communication Systems, Aug. 2013, pp. 1-5.

[28] _ - "Linear precoder design for doubly correlated partially coherent fading MIMO channels," IEEE Wireless Commun. Lett., vol. 13, no. 7, pp. 3621-3635, Jul. 2014.

[29] S. G. Srinivasan and M. K. Varanasi, "Constellation design for the noncoherent MIMO rayleigh-fading channel at general SNR," IEEE Trans. Inf. Theory, vol. 53, no. 4, pp. 1572-1584, Apr. 2007.

[30] A. Yadav et al., "Partially coherent constellation design and bit-mapping with coding for correlated fading channels," IEEE Trans. Commun., vol. 61, no. 10 , pp. 4243-4255, Oct. 2013.

[31] N. Serafimovski et al., "Fractional bit encoded spatial modulation (FBESM)," IEEE Commun. Lett., vol. 14, no. 5, pp. 429-431, May 2010.

[32] Y. Yang and S. Aissa, "Information guided channel hopping with an arbitrary number of transmit antennas," IEEE Commun. Lett., vol. 16, no. 10 , pp. $1552-1555$, Oct. 2012.

[33] M. Maleki and H. R. Bahrami, "Adaptive space modulation with partial csit in spatially correlated fading channels," IEEE Trans. Veh. Technol., vol. 63, no. 7, pp. 3184-3195, Sep. 2014.

[34] A. Stavridis et al., "Performance analysis of multistream receive spatial modulation in the MIMO broadcast channel," IEEE Transactions on Wireless Communications, vol. 15, no. 3, pp. 1808-1820, Mar. 2016.

[35] R. Zhang et al., "Error probability and capacity analysis of generalised pre-coding aided spatial modulation," IEEE Trans. Wireless Commun., vol. 14, no. 1, pp. 364-375, Jan. 2015.

[36] D. Tse and P. Viswanath, Fundamentals of Wireless Communication. Cambridge, UK: Cambridge University Press, 2005.

[37] Q.-T. Ngo et al., "General minimum euclidean distance-based precoder for MIMO wireless systems," EURASIP J. Adv. Signal Process., vol 2013, no. 1, pp. 1-12, 2013.

[38] N. S. Perović et al., "Design of a simple phase precoder for generalized spatial modulation in LOS millimeter wave channels," in 11th Int. ITG Conf. on Systems, Communications and Coding (SCC 2017), 2017.

[39] N. Serafimovski et al., "Practical implementation of spatial modulation," IEEE Trans. Veh. Technol., vol. 62, no. 9, pp. 4511-4523, Nov. 2013.

[40] S. Guo et al., "On the mutual information and constellation design criterion of spatial modulation MIMO systems," in 2014 IEEE Int. Conf. on Communication Systems (ICCS). IEEE, 2014, pp. 487-491.

[41] R. Mesleh et al., "Quadrature spatial modulation," IEEE Trans. Veh. Technol., vol. 64, no. 6, pp. 2738-2742, Jun. 2015. 